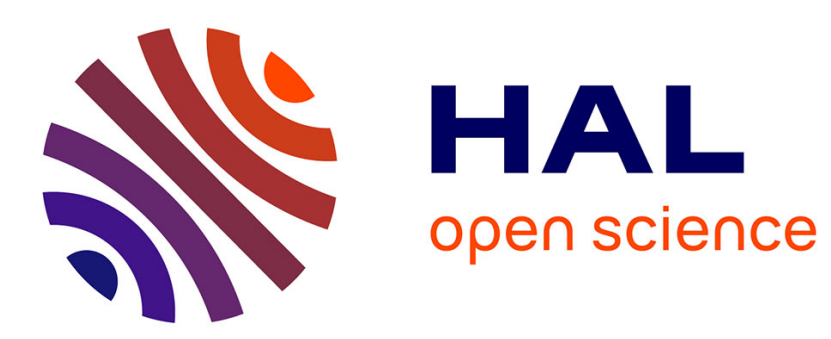

\title{
The Principle of Minimum Differentiation Revisited: Return of the Median Voter
}

\author{
Nobuyuki Hanaki, Emily Tanimura, Nicolaas J Vriend
}

\section{To cite this version:}

Nobuyuki Hanaki, Emily Tanimura, Nicolaas J Vriend. The Principle of Minimum Differentiation Revisited: Return of the Median Voter. Journal of Economic Behavior and Organization, 2019, 157, pp.145-170. 10.1016/j.jebo.2017.12.014 . hal-01714582

\section{HAL Id: hal-01714582 \\ https://hal.science/hal-01714582}

Submitted on 21 Feb 2018

HAL is a multi-disciplinary open access archive for the deposit and dissemination of scientific research documents, whether they are published or not. The documents may come from teaching and research institutions in France or abroad, or from public or private research centers.
L'archive ouverte pluridisciplinaire HAL, est destinée au dépôt et à la diffusion de documents scientifiques de niveau recherche, publiés ou non, émanant des établissements d'enseignement et de recherche français ou étrangers, des laboratoires publics ou privés. 


\title{
The Principle of Minimum Differentiation Revisited: Return of the Median Voter*
}

\author{
Nobuyuki Hanaki ${ }^{\dagger} \quad$ Emily Tanimura ${ }^{\ddagger} \quad$ Nicolaas J. Vriend ${ }^{\S}$
}

September 11, 2017

\begin{abstract}
We study a linear location model (Hotelling, 1929) in which $n$ (with $n \geq 2$ ) boundedly rational players follow (noisy) myopic best-reply behavior. We show through numerical and mathematical analysis that such players spend almost all the time clustered together near the center, re-establishing Hotelling's "Principle of Minimum Differentiation" that had been discredited by equilibrium analyses. Thus, our analysis of the best-response dynamics shows that when considering e.g. market dynamics as well as their policy and welfare implications, it may be important to look beyond equilibrium analyses.
\end{abstract}

JEL Classification: C72, D72, L13, R30

Keywords: Hotelling location model, Principle of Minimum Differentiation, Nash equilibrium, Best-response dynamics, Stochastic stability, Invariant measures

\section{Introduction}

Hotelling (1929) showed in a linear location model, in which two players independently choose a location, with their payoffs depending on the distance from

*We thank participants of the WEHIA meeting in Nice for comments and discussions. Part of this research has benefited from financial support from the French government managed by l'Agence Nationale de la Recherche under Investissements d'Avenir $U C A^{J E D I}$ (ANR-15-IDEX01). In particular, we thank the UCAinACTION project. All errors are our own.

†Université Côte d'Azur, CNRS, GREDEG. < Nobuyuki.HANAKI@unice.fr>

†Centre d'économie de la Sorbonne, Université Paris 1. <emily.tanimura@univ-paris1.fr>

$\S$ Corresponding author: School of Economics and Finance, Queen Mary University of London. $<$ n.vriend@qmul.ac.uk> 
individuals distributed on the line, that both players will locate together in the center. This result led to the notion of the "Principle of Minimum Differentiation".

Hotelling's model, where individuals have preferences over locations, provided a theoretical explanation for some casual observations that he reported about a widespread tendency for decision makers to choose only slight deviations from each other's location in the most diverse fields of competitive activity, even quite apart, as he put it, from what is typically called economic life. Besides geographical locations, he also discussed locations in product characteristics space, mentioning a "tremendous standardization of our furniture, our houses, our clothing, our automobiles and our education" (p. 54), as well as the choice of platforms by political parties in policy space.

Building on Hotelling's results, Black (1948) and Downs (1957) established the "Median Voter Theorem", i.e., that a majority voting rule will select the outcome preferred by the median voter. Also related is "Duverger's Law" (Duverger, 1954), which suggests that with plurality voting, one should expect a two-party system. This has given rise to an extensive empirical and theoretical literature (see e.g. Riker (1982), Rowley (1984), Osborne (1995), Grofman (2004), and many others). In this paper, we will go back to Hotelling (1929), and focus on one single aspect of this literature: The Principle of Minimum Differentiation.

Hotelling's result that in equilibrium the players will locate together in the center turns out to be correct for the special case of $n=2$ players. As we will sketch in Section 2, equilibrium analyses have made clear that the Principle of Minimum Differentiation is not robust to changing the number of players $n$ in the basic location model to any number $n>2$. In fact, for any $n>2$, the Nash equilibria look almost the opposite of what is described by the Principle of Minimum Differentiation, with all players spread out relatively evenly, and strict bounds on the spatial differences between the players. 
Experimental evidence for Hotelling's location game in the laboratory with $n=3$ (Collins and Sherstyuk, 2000) and $n=4$ (Huck, Müller, and Vriend, 2002) suggests that one may expect many non-equilibrium outcomes in this game, with in particular more choices near the center than predicted by the theory. ${ }^{1}$

Given the apparently widespread centripetal tendency of decision makers in a range of different settings, as argued e.g. by Hotelling (1929), typically also in cases where there are more than two decision makers, and given also the experimental evidence for the basic Hotelling game, the game-theoretic analysis of the basic location game raises the important question as to how one may explain such a centripetal tendency. In the industrial organization literature, Palma, Ginsburgh, Papageorgiou, and Thisse (1985) showed that one way to restore the Principle of Minimum Differentiation is to assume that there is sufficient heterogeneity in consumers' tastes combined with uncertainty by the firms about these preferences. Another approach is to consider a different equilibrium concept. Shino and Kawasaki (2012) characterize the farsighted stable set of the Hotelling game, and show that this set contains location profiles that reflect the minimum differentiation.

In this paper, we will follow an alternative approach. Instead of an equilibrium analysis, we will analyze the dynamics of myopic best-response behavior. We will show, through numerical as well as formal, mathematical analysis, that the players will be located almost all the time close to the center if their location choices are governed by noisy, myopic best-responses to the other players. We show for $n \geq 3$ that players spend significantly much more time in locations that are closer to the center (and close to each other) than predicted by the Nash equilibria. What is more, we show that by refining the discrete space (thus approximating a continuous space), we can get the players located arbitrarily close to the center almost all the time, catering essentially just to the median voter. We also show that none of the

\footnotetext{
${ }^{1}$ See also Brown-Kruse, Cronshaw, and Schenk (1993) and Brown-Kruse and Schenk (2000) for related duopoly experiments.
} 
pure strategy Nash equilibria for any $n \geq 3$ is stochastically stable.

Thus, while the Principle of Minimum Differentiation is consistent with Nash equilibrium behavior only for $n=2$, to the extent that there may be a tendency for decision makers to choose (noisy) myopic best-responses, our analysis suggests a possible explanation as to why this popular notion is so ubiquitous, acquiring almost "folk wisdom" status, as well as for the empirical evidence this is based on.

Providing a possible explanation for some empirical phenomenon is not the only reason why our analysis may be interesting. That best-response dynamics may lead to outcomes that are so different from the Nash equilibrium analyses seems an intriguing feature of the Hotelling game from a theoretical point of view, and relates to the literature on best-response dynamics and Nash equilibria. See, e.g., Hofbauer (1995), Hopkins (1999), and Balkenborg, Hofbauer, and Kuzmics (2013).

The rest of the paper is organized as follows. Section 2 presents the basic Hotelling location model as well as a brief overview of the equilibrium analysis. Section 3 presents our numerical analysis, to gain some insight into the behavior of best-response dynamics in our model. We, then, formally characterize the long-run behavior for the case of $n=3$ in Section 4 , followed by the case of $n \geq 4$ in Section 5 . The welfare implications of our analysis are presented in Section 6, and Section 7 presents some concluding remarks.

\section{Equilibrium and minimum differentiation}

\subsection{Continuous location model}

In this section, we will give a brief overview of the various equilibria found in Hotelling's location game. We focus our attention on a basic, linear location model, which we will present for convenience as a model of spatial voting where the players are parties who choose a platform $x$ in a one-dimensional, continuous policy space 
(normalized to $[0,1]$ ). Following Eaton and Lipsey (1975), assume that no two parties can choose exactly the same location, with the minimum distance being $\delta$ (with $\delta$ close to 0 ). An infinite number of voters with unit mass, whose preferences are distributed uniformly over this space, vote for the player who are closest to their preferred location. The spatial distribution of voters is known to the players.

The payoff for a player from choosing location $x$ is simply the share of votes received at that location. Therefore, if the two nearest players to the left and to the right are located in $L(x)$ and $R(x)$ respectively, with $L(x)<x<R(x)$, the payoff of choosing location $x$ is given by:

$\pi(x \mid L(x), R(x))=\left\{\begin{array}{l}\frac{R(x)-L(x)}{2} \text { if } 0 \leq L(x)<R(x) \leq 1 \\ \left(x+\frac{R(x)-x}{2}\right) \text { if no player to the left of } x \text { and } R(x) \leq 1 \\ \left(1-x+\frac{x-L(x)}{2}\right) \text { if } 0 \leq L(x) \text { and no player to the right of } x\end{array}\right.$

Eaton and Lipsey (1975) characterized pure strategy Nash equilibria for games with any number of players but three. If $n=2$, the unique Nash equilibrium is when both players locate at $x=\frac{1}{2}$, giving a payoff $\pi=\frac{1}{2}$, as any other location would imply a lower payoff. For $n=3$ there is no Nash equilibrium in pure strategies. Any of the peripheral players will want to move in towards the interior player as much as possible, but that interior player will always want to jump out of this squeeze. We will turn our attention to mixed strategy equilibria in a moment, but first we will consider equilibria in pure strategies for $n>3$.

For $n=4$, the Nash equilibrium configuration has two players located at $\frac{1}{4}$ and the other two at $\frac{3}{4}$. Each player will get a payoff of $\frac{1}{4}$. When $n=5$, there will be three locations that will be occupied in equilibrium: one in the center (occupied by one player), and two peripheral ones equally distanced from the center, located at $\frac{1}{6}$ and $\frac{5}{6}$ respectively, and each occupied by two players. Note that this means that 
the interior player will get a payoff of $\frac{1}{3}$, whereas the four peripheral players will each get a payoff of $\frac{1}{6}$.

Eaton and Lipsey show that there is multiplicity of equilibria when there are more than 5 players (for any $n>5$ ). For $n=6$, one equilibrium configuration involves three locations, with $\frac{1}{6}, \frac{3}{6}$ and $\frac{5}{6}$ each occupied by two players, and each of them getting a payoff of $\frac{1}{6}$. An alternative equilibrium configuration involves the four locations $\frac{1}{8}, \frac{3}{8}, \frac{5}{8}$ and $\frac{7}{8}$, where the two peripheral locations are occupied by two players each and the two interior ones by one player each. In this case the two interior players will get a payoff of $\frac{1}{4}$ and the four peripheral ones a payoff of $\frac{1}{8}$. Between these two equilibria there is an infinite number of additional equilibria. Think of the second equilibrium as a stretched version of the first one. As the interval between the two peripheral locations gets gradually stretched, for any distance one can compute the required distance for the interior players such that no player has an incentive to deviate. The same logic, and hence multiplicity of equilibria, applies for any $n>6$.

Eaton and Lipsey present an informal proposition (for any $n \geq 6$ ) computing the bounds for the locations of the peripheral pairs of players, as well as for the vote shares of each of the players as a function of $n$. The upper bound for the distance from the boundary of the space to the leftmost (or rightmost) player is $\frac{1}{n}$. If the space left at the extreme by the peripheral player were greater than that, this player would get a payoff greater than $\frac{1}{n}$, and hence the average payoff of the other players must be strictly less than $\frac{1}{n}$, which means that at least one of them could profitably deviate to this space left by the peripheral player and get a payoff of at least $\frac{1}{n}$. Thus, in any equilibrium the distance between the leftmost and rightmost player will be at least $\frac{n-2}{n}$, which will approach 1 as $n$ increases. Within this range the players must be spread relatively evenly. Towards the two boundaries there must be a pair of peripheral players, as the outermost players always have an incentive to 
move towards their neighbours as far as possible. Interior players may be located either individually or in pairs. If they appear as a pair their distance will be $\delta$, but otherwise the upper bound for the intervals between players will be $\frac{2}{n}$. What is more, in equilibrium no player can get more votes than twice the number of votes for any of the other players.

We now consider mixed strategy Nash equilibria. Although no pure strategy equilibrium exists for $n=3$, there is a doubly symmetric mixed strategy Nash equilibrium characterized by Shaked (1982): All players independently choose locations $x$ (with $\frac{1}{4} \leq x \leq \frac{3}{4}$ ) with equal probabilities. Ewerhart (2014) characterized the set of mixed Nash equilibria for $n \geq 4$, showing that as $n$ increases this leads to a more dispersed distribution of individual locations. The distributions are sharply M-shaped, with most weight at locations at the periphery of the support interval. The support increases as $n$ increases, and for the reported values of $n$ exceeds that of the maximum distance between the peripheral players in the corresponding pure strategy Nash equilibria.

We can summarize these findings by noting that for any $n>2$ the equilibrium analyses seem to discredit the Principle of Minimum Differentiation as the locations chosen in the Nash equilibria are spread out considerably, essentially covering the entire space.

Besides varying the number of players $n$, a number of alternative variations of the basic model and their effect on the equilibrium predictions have been considered in the literature. For example, Eaton and Lipsey (1975) consider one-dimensional spaces without bounds, or two-dimensional spaces, showing that the Principle of Minimum Differentiation will normally not hold. Apart from considering other spatial dimensions, equilibrium locations may contradict the Principle also when extending the basic Hotelling game in other dimensions. For example, in industrial organization, d'Aspremont, Gabszewicz, and Thisse (1979) show that in a two- 
stage game where two firms choose their locations in the first stage, and then compete in terms of prices in the second stage, when the cost of transportation is quadratic for consumers, firms will locate in the opposite ends of the line to soften the competition in terms of prices. $^{2}$ Irmen and Thisse (1998) consider product differentiation in a multi-characteristics space, and show that firms will choose to maximize differentiation in some dominant (salient) characteristic while minimizing differentiation in the others. Similarly, in political economy, the Principle may not apply if political parties care not only about winning elections but are also ideologically motivated, i.e., they care about the policy actually implemented. Even with only two parties, in equilibrium we may see diverging platforms if there is sufficient uncertainty about the location of the median voter (Drouvelis, Saporiti, and Vriend, 2014).

\subsection{Discrete location variant}

For the remainder of the paper we will focus on a slight variation of the basic, linear location model sketched above. Instead of a continuous strategy space, we will consider a discrete space. We discretize the interval $[0,1]$ into $2 M+1$ equally spaced locations $x \in\{0,1, . ., M-1, M, M+1, \ldots, 2 M\}$, where $x=0$ and $x=2 M$ correspond to the two boundaries, 0 and 1 respectively, and $x=M$ is the median. Higher values of $M$ correspond to a finer discretization of the space. ${ }^{3}$ As before, the spatial distribution of voters (uniform with full support) is known to all $n$ players, and it will stay constant.

Each player can only occupy one of these discretized locations at any point in

\footnotetext{
${ }^{2}$ Matsumura, Matsushima, and Yamanori (2010) analyze the consequences of the evolutionary dynamics of two firms competing in the setting of d'Aspremont, Gabszewicz, and Thisse (1979), and show that such dynamics restore the minimum differentiation in that, under the unique stochastically stable equilibrium, the two firms will locate in the center and set their prices equal to their marginal costs.

${ }^{3}$ Note that this implies that the number of locations will be odd, ensuring that the space has a median, location $M$, that can actually be chosen by the players. Results for the numerical analysis of the best-response dynamics for even numbers of locations can be found in Appendix A.
} 
time. But any location $x$ can be selected by any of the players simultaneously. We say two players are exactly paired if they occupy the same location. If they occupy consecutive locations, we say they are non-paired. Voters will go for the nearest player. ${ }^{4}$ The payoff for a player from choosing location $x$ is simply the number of votes received at that location. When the number of players in location $x$ is $n_{x}$, and two nearest players to the left and to the right are located in $L(x)$ and $R(x)$ respectively, with $L(x)<x<R(x)$, the payoff of choosing location $x$ is given by:

$$
\pi\left(x \mid n_{x}, L(x), R(x)\right)=\left\{\begin{array}{l}
\frac{1}{n_{x}} \frac{R(x)-L(x)}{2} \text { if } 0 \leq L(x)<R(x) \leq 2 M \\
\frac{1}{n_{x}}\left(x+\frac{R(x)-x}{2}\right) \text { if no player to the left of } x \text { and } R(x) \leq 2 M \\
\frac{1}{n_{x}}\left(2 M-x+\frac{x-L(x)}{2}\right) \text { if } 0 \leq L(x) \text { and no player to the right of } x
\end{array}\right.
$$

The (pure strategy) Nash equilibria in this discrete variant have the following properties: $:^{5}$

(i) The peripheral players are always (exactly or non-exactly) paired.

(ii) The interior players can be alone or in pairs of two.

(iii) If two players are exactly paired the size of their left market is equal to the size of their right market.

\footnotetext{
${ }^{4}$ When a voter finds that the best option(s) to the left and the best option(s) to the right are equally distant, they choose between going left or right by a fair coin toss. If, then, there is more than one player at location $x$, votes will be equally divided among the players located there.

${ }^{5}$ Most of these properties can be established by simply adapting the analysis of Eaton and Lipsey to a discrete setting. We will however provide a short proof of properties (iii) and (iv), which are not mentioned in the analysis of Eaton and Lipsey. For Property (iii), suppose that the two exactly paired players are located at $m$. Let $l$ and $r$ be the location of the players closest to their left and to their right. At $m$, the exactly paired players earn the payoff $\frac{(r-m)+(m-l)}{4}$. Now suppose for example that the left market is greater than the right market: $m-l>r-m$. Then, if a player unilaterally deviates, relocating at $l+1$, he earns $\frac{m-l}{2}>\frac{(r-m)+(m-l)}{4}$. This contradicts the Nash equilibrium property. Similarly, if the right side of the market was larger, players would have an incentive to deviate to that side. To prove property (iv), let the non-exactly paired players be located at $m$ and $m+1$, and let $l$ and $r$ be the closest location to the left of $m$ and to the right of $m+1$. The payoff of the player at $m$ is $\frac{m+1-l}{2}$. The payoff of the player at $m+1$ is $\frac{r-m}{2}$. Suppose for example that $\frac{r-m}{2} \geq \frac{m+1-l}{2}+1$, then by relocating at $m+2$ the player at $m$ could earn $\frac{r-(m+1)}{2}>\frac{m+1-l}{2}$, contradicting the equilibrium property.
} 
(iv) If two players are non-exactly paired, the difference in size between their markets is at most one.

(v) No player can have a market size greater than twice the size of another player.

Thus, the discreteness of the strategy space and the opportunity for more than one player to select the same location induce some slight changes to the equilibria sketched in Section 2 for the continuous model of Eaton and Lipsey, but the main qualitative features, with all Nash equilibria for any $n>2$ looking strongly at odds with the Principle of Minimum Differentiation, are not affected. ${ }^{6}$

\section{Best-response dynamics: numerical analysis}

Before turning to the formal, mathematical analysis of the dynamics of best-response behavior in the Hotelling model in Sections 4 and 5, where we characterize the invariant distribution of the players in the long run, in this section we will present a numerical analysis of the dynamics of the system. We will report on the dynamics for values of $M \in\{1500,15,000,150,000\}$, and values of $n \in\{3,4,5,6,7,8\} .^{7} 8$

In each period, all players simultaneously decide where to locate themselves, except in the very first period when they are all located randomly. We consider noisy myopic best-replies. Each player chooses a myopic best-reply with probability $1-\epsilon$, and chooses a location uniformly randomly with probability $\epsilon$. We report

\footnotetext{
${ }^{6}$ The minor differences are illustrated e.g. in Huck, Müller, and Vriend (2002), where we see that with $n=4$ in equilibrium the paired players may stay either in the same location or in two neighboring ones. And of course, as noted already, in a continuous strategy space there is an infinite number of Nash equilibria for any $n>5$, and thus any discretization will be able to relate to only a subset of these.

${ }^{7}$ We chose the values of $M$ so that the number of intervals will be a multiple of 60 , accommodating many of the Nash equilibria in the discretized system for the numbers of players $n$ that we consider.

${ }^{8}$ Note that the state space in our model (depending on the position of $n$ players in $2 M+1$ locations) is ${ }_{2 M+1} H_{n}={ }_{2 M+n} C_{n}=(2 M+n) ! /(2 M ! n !)$, with all elements being non-zero. Given this size of the state space, an eigenvalue analysis of such a transition matrix seems computationally unattractive.
} 
the results for $\epsilon=0.001$. When myopically best-replying, each player takes the positions of the other players in the previous period as given and selects a position that maximizes his payoff. When there are multiple such locations, one of them will be chosen (uniform) randomly. ${ }^{9}$

Best-response dynamics are related to a broad class of learning dynamics and evolutionary dynamics (see, e.g., Hopkins, 1999). The underlying idea of considering such a plausible class of dynamics is to shed some light on the question as to whether one should expect (boundedly rational) players to play a Nash equilibrium. If bestresponse dynamics converge, it can only be at a strict Nash equilibrium. But if they exhibit endless cycling, one question to consider will be where the system spends most of the time.

As we saw above, the location game is characterized by a multiplicity of equilibria (for any $n>5$ in pure strategies, and any $n>3$ if we consider mixed strategies too), and all these Nash equilibria are weak, as there are always some players who are indifferent between their equilibrium locations and some alternative locations. ${ }^{10}$ As players choose randomly among their best-replies, for any equilibrium there may be some individual players who move out of their equilibrium location, which may render these equilibria unstable as other players may be affected. In addition to this effect of the equilibria being weak, the best-response dynamics that we consider are characterized by a small amount of noise. This prevents the system getting stuck in simple periodic trajectories. ${ }^{11}$ The presence of noise ensures that the location dynamics will be an ergodic Markov chain. It is then well known that their long run

\footnotetext{
${ }^{9}$ We also considered a number of variants of the model. Variants with even numbers of locations, with different noise levels, with sequential moves, where in each period one randomly chosen player decides where to locate himself, and with inertia, in which players for whom the current location is part of their best-response correspondence will stay put. Results for these variants, documenting the robustness of our main results, can be found in Appendix A.

${ }^{10}$ While this is trivially true for any mixed Nash equilibrium, it is also the case in the pure strategy equilibria for any $n>3$, where there are always some players who could deviate from an equilibrium location to any two locations that are between two other players without loss of payoffs.

${ }^{11}$ It is also effective in the variant with inertia (where players stay put in case of indifference).
} 
behavior will be described by an invariant distribution on the states that is reached regardless of the initial conditions of the system.

The question, then, is what outcomes one should expect in these simple linear location models when players follow a noisy myopic best-reply. Although one should not expect perfect convergence to a weak Nash equilibrium, one possible outcome would be that they spend most of the time near or approximating Nash equilibrium locations.

Before we will turn to an examination of some statistics of the system later in this section, we start our numerical analysis with a relatively close-up look at a number of representative runs of the model, examining how individual players move around from period to period. This will also provide some insights that may be helpful in our formal, mathematical analysis in Sections 4 and 5.

Figure 1 shows some representative snapshots of locations chosen by all $n$ players over a 1000 period interval for $M=1500$, and $n=3,4,5,6,7,8$, with $\epsilon=0.001$. Each location chosen by each player in each period is represented by a dot. The 1000 periods were taken after 10,000,000 periods had passed from the beginning of each run to reduce the possible effects of the initial, random allocations. Time is shown on the horizontal axis, while the vertical axis shows the locations. The left-hand side column shows the 1000 locations near the center for the graphs for $n=3,4,6$ and 8 , while the right-hand side column shows the same runs, but now with the vertical axis zoomed into the most relevant parts of the strategy space for each graph to optimize the display of the locations.

Figure 1 reveals some interesting features. For $n=3$, the players are basically staying within a small set of locations around the center. For $n \geq 4$, we can see more clearly how the dynamics are dominated by waves of outward expansion of two clusters of players that are equally distanced from the center and located in the opposite sides of the center, alternated with waves of single clusters slowly moving 

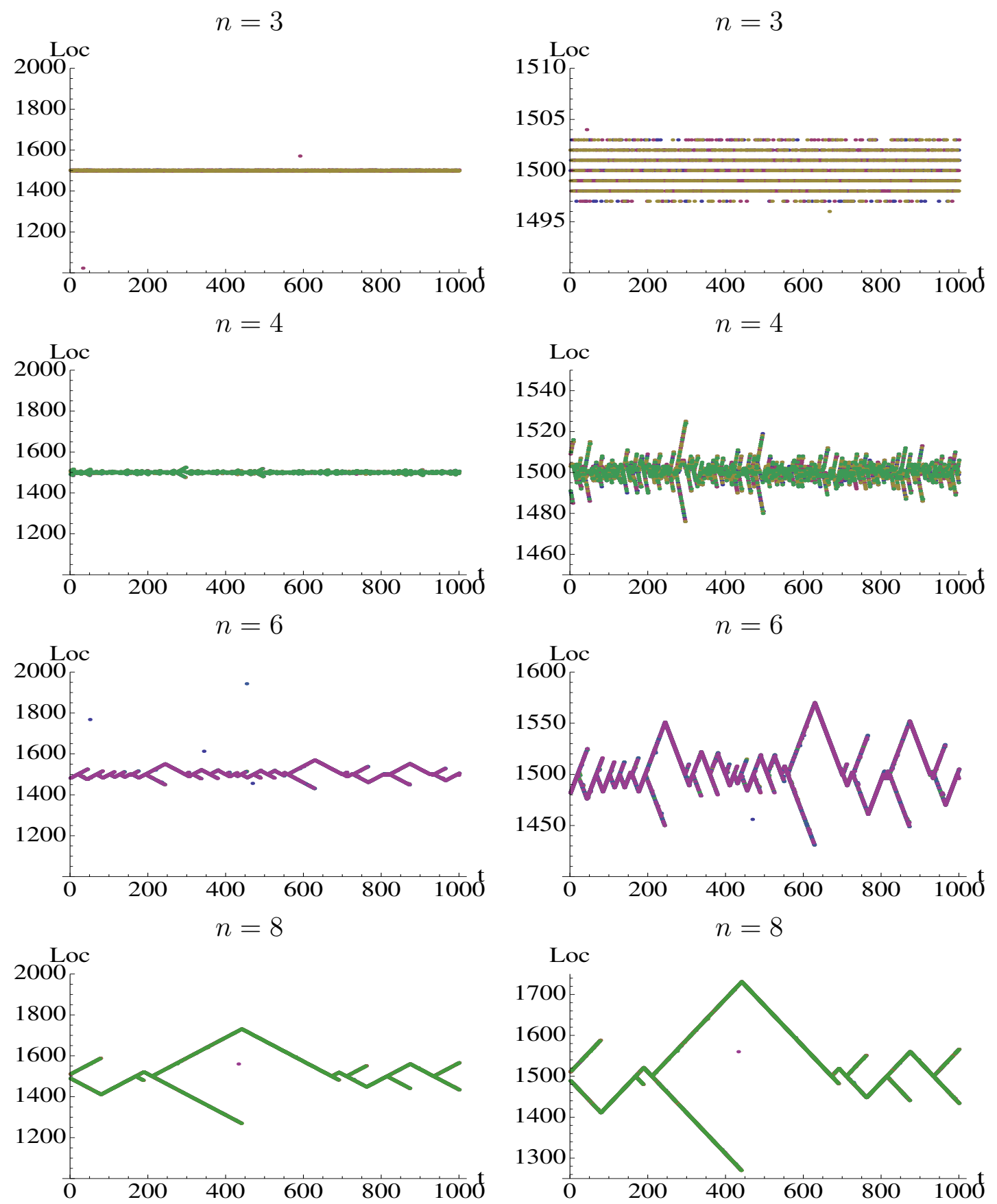

Figure 1: Locations over 1000 period interval for $M=1500, n=3,4,6,8$, with $\epsilon=0.001$ 
inward to the center. While riding the outward waves, when it comes to choosing a best-response all players are indifferent between these two clusters, as long as there is at least one other player in their current cluster. As a result, the numbers involved within each of the clusters may vary from period to period, and at some point it will happen that all the players locate themselves in the same cluster, and hence in the same half of the strategy space. The single cluster, then, starts moving step-by-step towards the center. ${ }^{12}$ Once the cluster reaches the center, there are two possibilities. Either all players stay together, moving to either $M-1$ or to $M+1$ before returning to $M$. Or the cluster splits into two, with some players moving to $M-1$ and some others to $M+1$, followed by a step-by-step outwards movement of both clusters, with varying memberships, until all players happen to choose the same side of the strategy space again. To start these waves all that is needed is that all players are located in the same half of the strategy space. ${ }^{13}$

Note that in none of the snapshots do we see convergence to the corresponding Nash equilibrium. Instead, we tend to see the wrong clustering at the wrong locations and moving into the wrong direction. At first sight, the most likely candidate to be reached by best-response dynamics is the Nash equilibrium for the case of $n=4$, as it consists of exactly two clusters of two players at locations $\frac{M}{2}$ and $\frac{3 M}{2}$. Taking a closer look at this case provides an interesting illustration for the general lack of convergence to equilibrium. Note that, for any $n$, the farthest the outward-moving waves can reach is a distance $\frac{M}{2}$ from the center. The clusters are shattered at that point because players prefer to move to anywhere between the two clusters rather than continue their outward movement. Thus, if this Nash equilibrium were reached, it would be shattered immediately. But as Figure 1 shows, the outward-moving clusters typically disintegrate already long

\footnotetext{
${ }^{12}$ Note that during their move to the center at every step all players select the same location. That is, there is minimum differentiation even when they are still on their way to the center.

${ }^{13}$ This takes, depending on $n$, typically only a few periods from a random initial allocation.
} 
before the equilibrium locations are reached. For other values of $n$ the dynamics look similar, even though the Nash equilibrium configurations are not characterized by two clusters. What is more, for any $n>4$ the Nash equilibria would require some players to move beyond the points where clusters would stop moving outwards and start disintegrating with some players moving back inwards.

Next, what about the long-run properties in terms of the average distances of players from the center for various $n$ ? How do they vary when we increase the number of locations, i.e, when we increase $M$ ? To answer such questions, we now turn to some statistics of these best-response dynamics, moving beyond these representative runs.

We analyze the myopic best-replies for $11 \times T$ periods where $T=10,000,000$. We drop, again, the data from the first $T$ periods to reduce the possible effects of the initial, random allocations, and keep the outcomes from the remaining $10 \times T$ periods. For a given run, for each period we compute the average distance of the $n$ players from the center, and we then check for that run the distances from the center below which this average distance is found $90 \%, 95 \%$ and $99 \%$ of the 100 million periods. Table 1 reports these distances for $n=3,4,5,6,7$ or 8 and for $M=1500$, 15, 000 and 150,000, with $\epsilon=0.001$ throughout. The reported mean distances (with the standard deviations in parentheses) are taken over 30 runs.

To compare with the outcomes of the noisy best-replies dynamics, Table 1 also reports the distances for the Nash equilibrium predictions that minimize the average distance from the center, i.e., the equilibria with as much clustering of players in the middle as possible. ${ }^{14}$

Table 1 shows that when $n=3$, regardless of the number of locations, the players are within distance 2 from the center almost all the time. Moving beyond $n=3$, the average distance from the center increases with the number of players $n$. This is true

\footnotetext{
${ }^{14}$ For $n=3$ this is based on the mixed strategy Nash equilibrium, and therefore we report the average distance below which the system will spend $90 \%$ of the time.
} 


\begin{tabular}{|c|c|c|c|c|c|c|}
\hline \multicolumn{7}{|c|}{$M=1500$} \\
\hline & $\mathrm{n}=3$ & $\mathrm{n}=4$ & $\mathrm{n}=5$ & $\mathrm{n}=6$ & $\mathrm{n}=7$ & $\mathrm{n}=8$ \\
\hline \multirow[t]{2}{*}{$90 \%$} & 2.0 & 8.0 & 30.0 & 66.0 & 123.7 & 203.7 \\
\hline & $(0.0)$ & $(0.0)$ & $(0.0)$ & $(0.0)$ & $(0.5)$ & $(0.6)$ \\
\hline \multirow[t]{2}{*}{$95 \%$} & 2.0 & 10.0 & 40.0 & 86.9 & 161.3 & 265.5 \\
\hline & $(0.0)$ & $(0.0)$ & $(0.0)$ & $(0.3)$ & $(0.5)$ & $(0.9)$ \\
\hline \multirow[t]{2}{*}{$99 \%$} & 2.0 & 15.0 & 65.9 & 139.9 & 247.4 & 409.3 \\
\hline & $(0.0)$ & $(0.0)$ & $(0.4)$ & $(0.3)$ & $(0.8)$ & $(1.4)$ \\
\hline \multirow[t]{3}{*}{$\mathrm{NE}$} & 539 & 750 & 800 & 667 & 804 & 750 \\
\hline & \multicolumn{5}{|c|}{$M=15,000$} & \\
\hline & $\mathrm{n}=3$ & $\mathrm{n}=4$ & $\mathrm{n}=5$ & $\mathrm{n}=6$ & $\mathrm{n}=7$ & $\mathrm{n}=8$ \\
\hline \multirow[t]{2}{*}{$90 \%$} & 2.0 & 8.0 & 30.0 & 66.1 & 124.1 & 204.6 \\
\hline & $(0.0)$ & $(0.0)$ & $(0.0)$ & $(0.3)$ & $(0.4)$ & $(0.6)$ \\
\hline \multirow[t]{2}{*}{$95 \%$} & 2.0 & 10.0 & 40.0 & 87.3 & 164.2 & 271.1 \\
\hline & $(0.0)$ & $(0.0)$ & $(0.0)$ & $(0.5)$ & $(0.4)$ & $(0.9)$ \\
\hline \multirow[t]{2}{*}{$99 \%$} & 2.0 & 15.0 & 67.2 & 152.0 & 291.5 & 481.9 \\
\hline & $(0.0)$ & $(0.0)$ & $(0.4)$ & $(0.5)$ & (1.4) & $(2.4)$ \\
\hline \multirow[t]{3}{*}{$\mathrm{NE}$} & 5392 & 7500 & 8000 & 6667 & 8036 & 7500 \\
\hline & \multicolumn{5}{|c|}{$M=150,000$} & \\
\hline & $\mathrm{n}=3$ & $\mathrm{n}=4$ & $\mathrm{n}=5$ & $\mathrm{n}=6$ & $\mathrm{n}=7$ & $\mathrm{n}=8$ \\
\hline \multirow[t]{2}{*}{$90 \%$} & 2.0 & 8.0 & 30.0 & 66.1 & 124.2 & 204.7 \\
\hline & $(0.0)$ & $(0.0)$ & $(0.0)$ & $(0.3)$ & $(0.4)$ & $(0.6)$ \\
\hline \multirow[t]{2}{*}{$95 \%$} & 2.0 & 10.0 & 40.0 & 87.5 & 164.6 & 271.9 \\
\hline & $(0.0)$ & $(0.0)$ & $(0.0)$ & $(0.5)$ & $(0.5)$ & $(0.8)$ \\
\hline \multirow[t]{2}{*}{$99 \%$} & 2.0 & 15.0 & 67.9 & 154.0 & 302.0 & 525.6 \\
\hline & $(0.0)$ & $(0.0)$ & $(0.3)$ & $(0.5)$ & $(1.4)$ & $(3.8)$ \\
\hline $\mathrm{NE}$ & 53,914 & 75,000 & 80,000 & 66,667 & 80,357 & 75,000 \\
\hline
\end{tabular}

Table 1: Mean distances from the center (with standard deviations in parentheses), and NE distances, for $M=1500,15,000,150,000, n=3,4,5,6,7,8$, with $\epsilon=0.001$ 
for all values of $M$. Focussing on $M$, we note that the average distance below which the system spends time increases only very slightly as we move from $M=1500$ to $M=15,000$, and to $M=150,000$, with these increases being detectable only for larger values of $n$. Thus, for example, for $M=1500$ and $n=8$, we see that the distance from the center below which the system (i.e., the average distance of the $n$ players in the period concerned) spends $95 \%$ of the time is 265.5 , and this increases to only 271.9 when we increase the number of locations to 150,000 (a hundredfold increase)

We can compare this with how the average distances in the Nash equilibria increase with the number of locations. As we see in Table 1, the predictions of the Nash equilibria are not affected by the refinements of the strategy space in the sense that they are simply scaled up proportionally with the number of locations and hence stay away from the median at a constant relative distance. For example, the average distances from the center for $n=4$ are 750, 7500, and 75,000 for $M=1500$, 15,000 and 150,000, respectively. ${ }^{15}$

Table 1 also reflects the fact that as $n$ increases, the average distance of the Nash equilibrium that is closest to the center is essentially constant. ${ }^{16}$ As $n$ increases the peripheral players get closer to the boundaries, but this is exactly offset by the most central ones getting closer to the center, and thus the average distance stays constant. A good example of this is a comparison of the average distance for the Nash equilibria reported for $n=4$ and 8 .

We can conclude that Table 1 confirms what we saw in Figure 1, i.e. that the best-response dynamics do not converge to the Nash equilibria. ${ }^{17}$ Our numerical analysis also shows that for any $n>2$, as $M$, and hence the number of locations,

\footnotetext{
${ }^{15}$ If, for $n=3$, we consider the $99 \%$ criterion instead of the $90 \%$ reported in the Table, these will be 652,6521 , and 65,213 , for $M=1500,15,000$, and 150,000 , respectively.

${ }^{16}$ Apart from some minor variances as different values of $n$ lead to slightly different types of Nash equilibrium configurations.

${ }^{17}$ That the best-response dynamics lead to outcomes that are in some sense the 'opposite' of the Nash equilibrium predictions was also observed in Pancs and Vriend (2007).
} 
increases, the players tend to spend their time farther and farther away from the Nash equilibrium predictions and within smaller and smaller regions around the center. In other words, as we refine the discrete strategy space, approximating a continuous strategy space, we can get the players locating arbitrarily close to the median when they follow best-response dynamics.

Thus, the best-response dynamics seem to restore something close to the Principle of Minimum Differentiation and as a result, the preferences of the median voter will tend to rule.

\section{Long run behavior: analyzing the invariant measure with three players}

With the numerical analysis presented in Section 3 in mind, in this section and in Section 5, we characterize the long-run properties of the system. For any number of locations $M$ and any level of noise $\epsilon$, the long run behavior of the location dynamics is described by the invariant distribution $\mu_{M, \epsilon}$ of an ergodic Markov chain. The dynamics that we observe in the numerical analysis after a sufficiently large number of time steps, are in fact sample paths from the distribution $\mu_{M, \epsilon}$. Our analytical results concerning the behavior of the location dynamics are based on an asymptotic analysis (w.r.t. $M$ and $\epsilon$ ) of the invariant distribution $\mu_{M, \epsilon}$. In other words, we study this distribution when the number of locations tends to infinity and/or the level of noise is driven to zero. The asymptotic analysis is relevant for understanding the behavior of the dynamics when the number of locations is finite but large and for positive but small levels of noise, which is precisely the setting we are interested in here.

For the case of $n=3$ players analyzed in this section, we will let the number of locations $M$ be fixed and drive noise down to zero, as is a standard approach in 
the equilibrium selection literature. The states in which the invariant measure is concentrated as noise goes to zero are referred to as the stochastically stable states, and correspond to outcomes that remain stable when players make errors with a small probability.

In the remainder of this section we will characterize the stochastically stable steady states for the case of three players. For the case of four or more players, presented in Section 5, we will follow a somewhat different approach, explained in that section.

\subsection{Absorbing classes}

It is well known that when noise is driven down to zero, the invariant distribution will concentrate on some union of absorbing classes. A set of states is an absorbing class if we remain within the class under best-replies without noise $(\epsilon=0)$. Thus, we begin by characterizing the absorbing classes of our system.

Recall that we consider a system where the center is unique and denoted by $M$. The leftmost location is then 0 and the rightmost location $2 M$. Let $(a, b, c)$ denote the locations of the players, adopting the convention that $a \leq b \leq M<c$. By symmetry, we consider, without loss of generality, the case where two players are to the left of the center and the third player on the other side. ${ }^{18}$ The number of positions to the left of $a$ is then $a$ and the number of positions to the right of $c$ is $2 M-c$. If $a>2 M-c$ it is preferable to locate to the left of $a$ than to the right of $c$ and conversely. If $a=2 M-c$ these two choices are indifferent. We will also consider the distance between the peripheral, i.e. the most extremely located, players, which under the above assumptions is the length of the interval $[a, c]$, i.e., $c-a$. Also, $a$ and $2 M-c$ are the distances of the peripheral players to the boundaries. For given $M$, when $a$ and $2 M-c$ increase, the distance to the center decreases. We will denote

\footnotetext{
${ }^{18}$ The case where all players are on the same side will be treated separately.
} 
the set of best-replies to $(a, b, c)$ by $B R(a, b, c)$. We will denote by $B R^{l}, B R^{m}, B R^{r}$ the best-responses of the leftmost, middle and rightmost player respectively. We denote by $B R_{t}^{l}, B R_{t}^{m}, B R_{t}^{r}$ the $t$-th best-response of the leftmost etc. player. ${ }^{19} \mathrm{We}$ denote by $B R_{t}(a, b, c)$, or sometimes for convenience simply $B R_{t}$, the locations of the players after $t$ periods, determined by best-replies, starting from $(a, b, c)$. Bestreplies may be non-unique. Note that we will also order the best-reply to $(a, b, c)$ from the smallest to the largest element. Thus if $B R(a, b, c)=a^{\prime}, b^{\prime}, c^{\prime}$, we have $a^{\prime} \leq b^{\prime} \leq c^{\prime}$ but generally we do not have $\left(B R^{l}, B R^{m}, B R^{r}\right)=\left(a^{\prime}, b^{\prime}, c^{\prime}\right)$. The following proposition characterizes the absorbing classes of the system.

Proposition 1 Let $C=\{(M-3, M-1, M+1),(M-1, M+1, M+3),(M, M+$ $2, M+2),(M, M-2, M-2),(M-1, M+1, M+1),(M-1, M-1, M+1),(M-$ $2, M, M+2)$,$\} and C_{k}=\{(M-k, M-k, M+k-1),(M-k+1, M+k, M+k)\}$. Then, $C$ is an absorbing class, and $C_{k}$ is an absorbing class for $1 \leq k \leq K$, where $K=: \max \left\{k \mid 2 M-(M+k-1)>\frac{2 k-1}{2}\right\}$.

Proof: Immediate verification. The condition on $k$ ensures that the players on the left will prefer location $M+k$ to an interior location.

Although it is possible to show that $C$ and $\left(C_{k}\right)_{k=1}^{k=K}$ are the only absorbing classes, we will not need this result. In fact, in our analysis we will focus on the following union of absorbing classes: Define $S=C \bigcup C_{1} \bigcup C_{2}$.

\subsection{Basins of attraction}

If an element that is not in the absorbing class $S$ will eventually lead to $S$ by best replies with probability one, it is said to belong to the basin of attraction of $S$. The basins of attraction of the absorbing classes play an important role in determining

\footnotetext{
${ }^{19}$ Note that $B R_{t}^{l}$ refers to the $t$-th best-reply of the leftmost player at time $t-1$, which is not necessarily the leftmost player in the initial configuration.
} 
their stochastic stability. We recall that the basin of attraction $B(S)$ of an absorbing class $S$ is: $B(S)=\left\{z \mid \operatorname{Prob}\left(\exists T\right.\right.$ st $\left.\left.\left.B R_{t}(z) \in S \forall t>T\right)=1\right\}\right)$.

As the following two lemmas show, a large subset of configurations is in the basin of attraction $B(S)$ of the union of absorbing classes $S$ defined above.

Lemma 1 Any configuration $(a, b, c)$ where $a \leq b \leq c \leq M$ (or $M \leq a \leq b \leq c)$, i.e. with all players on the same side of the center, is in the basin of attraction of $S$.

Proof: We make explicit the possible BR sequences. If $a \leq b<c \leq M$, $B R(a, b, c)=(b+1, c+1,+c+1)$. If $b<2 M-c-2, B R_{2}=(c+2, c+2, c+2)$, which is considered below. If $b \geq 2 M-c-2$, which is only possible if $c=M$ and $b \in\{M-1, M-2\}$, these cases correspond to $B R \in\{(M, M+1, M+1),(M-$ $1, M+1, M+1)\}$. The first is in $C_{1}$ and the second in $C$. If $a \leq b=c<M$, we get $(c+1, c+1, c+1),(c+2, c+2, c+2)$, and then all the way to $(M, M, M)$. The configuration $(M, M, M)$ leads either to $(M-1, M-1, M+1)$ or $(M-1, M+1, M+1)$, which are both in $C$ or to $(M-1, M-1, M-1)$ and then $(M, M, M)$ or similarly to $(M+1, M+1, M+1)$ and then $(M, M, M)$. In this case we cycle, but with positive probability we will eventually reach $C$ from $(M, M, M)$.

Lemma 2 Any configuration that lies within $\{\omega \mid \omega \in[M-3, M+3]\}-C_{3}$ is in the basin of attraction of $S$.

Proof: Given in Appendix B. It simply requires checking a number of configurations.

Combined, Lemmas 1 and 2 characterize the basin of attraction $B(S)$ of the union of absorbing classes $S$ that is the focus of our analysis. 


\subsection{Stochastically stable steady states}

To show that the invariant distribution will be concentrated on $S$, we will use the radius-coradius theorem of Ellison (2000). The main idea behind this theorem is to associate a cost to any path of transitions. Loosely speaking, this cost is proportional to the number of random events (i.e. choices that are not best replies) required on the path. The stochastic stability of a set is then determined by comparing the cost of leaving its basin of attraction and the cost of entering its basin of attraction from the outside.

The Hotelling model with noise lies within the general setting considered by Ellison (2000) since a player locates with probability $1-\epsilon$ on a best reply, and with probability $\epsilon$ uniformly at random. In the Hotelling model, we can define a cost function as follows: the cost of the transition from configuration $z_{1}$ to $z_{2}$ is $c\left(z_{1}, z_{2}\right)=3-m$, where $m$ is the number of players in $z_{2}$ who play a best reply to $z_{1}$. In other words, the cost of a transition is the number of random events it requires. The cost of a path $\left(z_{1}, \ldots, z_{t}\right)$ can then be defined as $\sum_{i=1}^{i=t-1} c\left(z_{i}, z_{i+1}\right)$. It can be verified that this cost function satisfies the properties required to use Ellison's radius-coradius theorem.

The crucial quantities defined by Ellison (2000) are the radius and coradius of $S, R(S)$ and $C R(S)$ respectively. The coradius of $S$ basically measures the number of random events required to reach $B(S)$ from outside $S$. The radius of a union of stochastically stable sets measures the random events required to leave $B(S)$ starting from $S$.

The main result in Ellison (2000) is that the invariant measure is concentrated on a union of absorbing classes such that the number of random events required to leave this set (the radius) is greater than the number of random events required to reach it from the outside (the coradius):

Theorem 1 (Ellison, 2000) In a model of evolution with noise, let $\Omega$ be a union of 
limit states. If $R(\Omega)>C R(\Omega)$ then the long run stochastically stable set is contained in $\Omega$.

We will use this theorem in order to show that the stochastically stable steady state is contained in $S=C \bigcup C_{1} \bigcup C_{2}$. We first compute the radius and coradius of $S$.

Proposition 2 Let $S=C \bigcup C_{1} \bigcup C_{2}$, then the coradius of $S, C R(S)=1$.

To see that a single random event is sufficient to enter the basin of attraction of $S$, consider some $C_{k}$ with $k>2$. To move from such a $C_{k}$ into $S$, it is sufficient that one player, the one who is alone on one side, relocates at random to the other side. $^{20}$

Proposition 3 The radius of $S, R(S)>1$.

We show that a single random event is not enough to leave the basin of attraction of $S$ with Lemma 3 .

Lemma 3 Take a configuration $s \in S$. Relocate one player. Call this configuration $\omega$. Then there exists $0<t<3$ such that $B R_{t}(\omega) \in(\{\omega \mid \omega \in[M-3, M+3]\}-$ $\left.C_{3}\right) \cup B(S)$.

Proof: Given in Appendix B. It simply requires checking a number of configurations.

Lemma 3 shows that after a single random event, we will return to $(\{\omega \mid \omega \in$ $\left.[M-3, M+3] C_{3}\right) \cup B(S)$, which by Lemma 2 is also in $B(S)$. Consequently, any path from $S$ to a configuration that is not in the basin of attraction of $S$ requires at least two random events, and hence the cost is strictly greater than 1 .

\footnotetext{
${ }^{20}$ Note that this applies to any configuration outside $B(S)$, as they are all of the two on one side versus one on the other side type.
} 
Combining Proposition 2 and 3 shows that the radius $R(S)$ is strictly greater than the coradius $C R(S)$, and together with the theorem in Ellison (2000) this gives us the main result for our analysis of the case of three players:

Corollary 1 The long run stochastically stable set is a subset of $S=C \bigcup C_{1} \cup C_{2}$. All configurations in $S$ are such that the average distance to the center is strictly smaller than 3, and for none of the players is the distance to the center greater than 3.

\section{Long run behavior: analyzing the invariant measure with four or more players}

We now move to the analysis of cases with four or more players. As we have seen in Section 3, there is a difference in the dynamics between the cases of $n=3$ and $n \geq 4$. For $n=3$, as observed in the numerical analysis, the players spend almost all their time at locations whose average distance from the center is strictly less than three. In fact, with only three players, in the absence of random noise, the central location belongs to an absorbing class. Thus, the typical distance to the center is bounded independently of the system size.

For $n \geq 4$, instead, there are repeated waves of two-sided expansion and onesided contraction centered around the middle. The distance of these waves from the center is typically very small compared to the number of locations, but it is not bounded independently of the latter. More precisely, when the number of locations increases, the maximal distance of the waves from the center also increases but at a speed that is some orders of magnitude smaller. Thus, as the number of locations increases, the sizes of the waves become negligible compared to the size of the system. To capture this intuition formally, we let the number of locations grow and impose some restrictions on the level of random noise compared to the number of locations. 
We then show that the invariant measure is concentrated on the center and on some absorbing classes close to the center.

Therefore, in the asymptotic framework presented in this section, for the case of $n=4$ we will use the fact that $M$ is large and we will study the behavior of $\mu_{M, \epsilon}$ driving $M$ to infinity and noise down to zero simultaneously, while imposing some conditions on the relative speed of convergence of these quantities. Although by doing so we lose the connection to the equilibrium selection approach, such an asymptotic analysis does capture the behavior of our system when the number of locations is large and the noise level small.

We first provide some notation needed to state our main result.

\subsection{Description and notation}

We denote by $N$ the $2 M+1$ locations indexed by $\{0,1,2, \ldots, M, M+1, \ldots, 2 M\}$. Let $\omega \in\{0,1,2, \ldots, M, M+1, \ldots, 2 M\}^{4}$ be a configuration, i.e. giving the locations of the four players. For convenience, we will write $\omega=(a * k, \ldots)$, when the configuration $\omega$ has $k(k \geq 3)$ players located at $a$.

We denote by $\bar{a}$ the configuration where all players are at location $a$. The distance between two locations $a$ and $b$ is $d(a, b)=|a-b|$. For a configuration $\omega=(a, b, c, d)$, we define the distance from the center $M$ as $d(\omega, M)=: \max _{i=a, b, c, d} d(i, M)$. Given a configuration $\omega$ at date $t$, the configuration at $t+1$ is given by the realization of the following random variables: (1) First, we determine the number of players who do not relocate at one of their best replies. This number is given by a binomial random variable $X_{t}=\operatorname{Bin}(\epsilon, 4)$. (2) Given the realization of $X_{t}$, we draw a variable $Y_{t}$ following a uniform law on all the subsets of $\{1,2,3,4\}$ of size $X_{t}$. If $X_{t}=1$, each player has probability $\frac{1}{4}$ of being selected for relocation. (3) Finally, for any element $e \in\{1,2,3,4\}$ such that $e \in Y_{t}$, we draw its location uniformly at random. Any player who is not drawn for random relocation, picks each of his best replies 
according to a uniform probability.

This description, where we draw first the number of uniformly relocated players, then their identity, then their location, is convenient for our proof and obviously equivalent to relocating each player uniformly at random with i.i.d. probability $\epsilon$. Let us also introduce some notation for some particular subsets of configurations that will be of interest. Let $A$ be the set of configurations where all players are on the same side (i.e. $\geq M$ or $\leq M)$. Analogous to the case of three players, we note that the classes consisting of the two states $\{((M-a) * 3, M+a-1),(M-a+1,(M+a) * 3)\}$, with the same restriction on $a$ as we saw for $k$ in Proposition 1, are absorbing classes for the dynamics. Let us denote by $\Omega_{A C}$ the set of such absorbing classes. Moreover, let $\Omega_{A C}^{l}$ be the subset of such classes for which $d(a, M)<l$, in other words absorbing classes containing configurations that are closer than $l$ to the center.

\subsection{The setting with an increasing number of locations and the main result}

In the case of $n=3$ players, and in the result in the previous subsection, the number of locations was fixed and the level of noise $\epsilon$ converged to zero asymptotically. In other words, we studied the behavior of the invariant distribution $\mu_{N, \epsilon}$, holding $N$ fixed and taking the noise $\epsilon$ down to zero. From now on, we will analyze the behavior of the invariant distribution $\mu_{N, \epsilon}$ when $N$ goes to infinity and $\epsilon$ goes down to zero. We will put some restrictions on the level of noise compared to the number of locations. To this effect, assume that the level of noise in a Hotelling model with $N$ locations is $\epsilon_{N} \cdot{ }^{21}$ The invariant measure $\mu_{N, \epsilon_{N}}$ will be denoted simply by $\mu^{N}$. Our main result characterizes the asymptotic behavior of $\mu^{N}$ under some assumptions on the speed at which $\epsilon_{N}$ goes down to zero as $N$ increases.

\footnotetext{
${ }^{21}$ Later on, when $N$ is fixed, for convenience we will omit the index $N$, writing simply $\epsilon$.
} 
Theorem 2 In the Hotelling model with $N$ locations, let $I_{N}=\left[M-l_{N}, M+l_{N}\right]$ be an interval centered at $M$, of length $2 l_{N}$ and let $\left(I_{N}\right)^{C}$ be the complement of the interval in $\{0, \ldots, 2 M\}$, that is $\left(I_{N}\right)^{C}=\{0, \ldots, 2 M\}-I$. Let $S_{N}$ be a subset of locations such that $\omega \in S_{N} \Longleftrightarrow \omega \in[M-3, M+3] \cup \Omega_{A C}^{l_{N} / 2} \subset I_{N}$. Let $B_{N}$ be any set of states $B_{N} \subset\left(I_{N}\right)^{C}$ such that $\operatorname{card}\left(B_{N}\right) \leq \operatorname{card}\left(S_{N}\right)$. Suppose that the Condition 1 below is verified, then $\lim _{N \rightarrow \infty} \frac{\mu^{N}\left(B_{N}\right)}{\mu^{N}\left(S_{N}\right)}=0$.

Condition 1 There exist constants $\alpha \in] 0, \frac{1}{7}\left[\right.$ and $\beta_{M}<\infty$ and $N_{0}$ such that for every $N \geq N_{0}, l_{N}=N^{\alpha}$ and the level of random noise $\epsilon_{N}$ verifies $\epsilon_{N}=N^{-\beta}$, where $\beta \in\left[1, \beta_{M}\right]$.

Theorem 2 states that when we consider a certain set of states $S_{N}$ included in a 'small' (some orders of magnitude smaller than $N$ ) interval around the center, $I_{N}$, any set containing the same number of locations as $S_{N}$ (or a smaller number) and that is located outside of the interval $I_{N}$, i.e. farther from the center, has an invariant measure that is vanishingly small compared to the measure of $S_{N}$ when the number of locations is large. In particular, it follows from this result that states close to the center have more weight than the Nash equilibrium, and also more weight than absorbing classes farther from the center.

How demanding are assumptions under which this asymptotic result holds? The most important restriction imposed by Condition 1 concerns the speed at which $\epsilon_{N}$ goes down to zero as the number of locations increases, since we must have $\epsilon_{N} \leq \frac{1}{N}$. The upper bound of the size of the interval $l_{N}$ is not a demanding condition in our context. Since we want to show that the invariant measure is concentrated close to the center, we are only interested in intervals $l_{N}$ that are small compared to the total number of locations $N$. The lower bound of the size of $l_{N}$ and the lower bound on the noise are not very restrictive. Together they guarantee that $\epsilon_{N}^{2} \geq p^{l_{N}}$ for any $0<p<1$ when $N$ is large. We should note that Condition 1 is a sufficient but not necessary condition. 


\subsection{Analysis of the dynamics of the system and intuition for our results}

Before turning to the proof of Theorem 2 itself, we will provide some intuition for the result based on the behavior of the system's dynamics. ${ }^{22}$ A central part of our proof is to show that starting from the center, we will return to it with high probability. As we have seen in the numerical analysis shown in Figure 1, starting from the center, in absence of random noise, the dynamics would be as follows. If two players locate at $M-1$ and two at $M+1$, we move farther away from the center. However, there is also a positive probability that all players locate on the same side. As we have established previously, the dynamics will then bring them back to the center. Similarly, in the next step, all players may locate on $M-2$ and $M+2$ but again, with positive probability, they may all end up on the same side. In each period, we can move farther away from the center but there is also a positive probability that all players end up on the same side. The probability that the latter does not occur in a long sequence of time steps is very small. ${ }^{23}$ This description of the dynamics ignores the possibility of random relocations. However, with high probability only few random relocations occur (as shown in Lemma 4 in Appendix C.1). This makes the dynamics somewhat more complex but not significantly different from what is described above.

An occasional random relocation may lead to various configurations within the interval I. However, we show (Lemma 5 in Appendix C.2) that the configurations we reach are always either contracting, in the sense that best replies bring us closer to the center, or, if the state is such that best replies can be farther away from the center with positive probability, there is also a positive probability that all players

\footnotetext{
${ }^{22}$ Note that in what follows and also in the Proofs we will give later, we will often write $I$ instead of $I_{N}$ and $I^{C}$ instead of $\left(I_{N}\right)^{C}$ in order to simplify notation when $N$ is fixed.

${ }^{23}$ The conditions we impose in Theorem 2 guarantee that the probability of not returning in this way is in fact smaller than the probability of a random relocation.
} 
end up on the same side. This is because such configurations involve indifference on the part of some players. The typical, but not unique, example of such a state is the one described previously where two players are at $M-k$ and two players at $M+k$. Thus, we can only move far away from the center if we pass through configurations of the second type a large number of times. However, each time we do, there is a positive probability that all players end up on the same side and the probability of avoiding this event for a long time is very small.

So far, we have ignored the possibility of entering one of the absorbing classes in $\Omega_{A C}^{l}$. This case is analyzed separately in Appendix C.3. If such a class is reached, we remain there for a long time. The most probable way to exit is with a single random relocation. When a single random relocation occurs, with positive probability we move to another absorbing class farther from the center, or, also with positive probability, we enter a state where all players are on the same side. Again, it is highly unlikely to have a long sequence of realizations where we move farther away from the center without an occurrence of an event where all players end up on the same side.

Finally, the proof also involves showing that with a single random relocation we can move back from any state to the center. This is the object of Appendix C.5.

We now turn to the proof of Theorem 2 itself. In what follows, we often do not need to establish exact values of quantities but only their order of magnitude with respect to $N$ when $N$ is large. We will thus use the notation $g(N)=O(f(N))$ if $0<\lim _{N \rightarrow \infty} \frac{f(N)}{g(N)}<\infty$. Moreover, from now on we will write $l$ for $l_{N}$ and $\epsilon$ for $\epsilon_{N}$.

Our proof will make use of the following property of the invariant distribution of a Markov chain: $\mu(S)=\mu(b) E[V(S, b, b)]$, where $V(S, b, b)$ is a random variable that counts the number of times that the process reaches an element in $S$ before it reaches $b$, starting from state $b .^{24}$

\footnotetext{
${ }^{24}$ See, e.g., Kemeny and Snell (1960).
} 
To bound the value of this expectation, we will bound, on the one hand the expected number of times we return to $S$ starting from $S$, and on the other hand the probability of reaching $S$ from a state in $I^{c}$. These two bounds are the object of the following two propositions:

Proposition 4 The probability of not returning to the set $S$ when starting from a configuration in $S$ is bounded above by $1-q=O\left(\frac{\epsilon}{l^{2}}\right)$.

Proposition 5 Let $b \in I^{C}$, the probability of reaching $S$ from $b$ without passing again through $b$ is bounded below by $\tilde{q}=O(\epsilon)$.

The event $V(S, b, b)=k$, requires first that we move from $b \in I^{C}$ to $S$ without passing again through $b$, which occurs with a probability greater than $\tilde{q}$, and then that starting from $S$, we return again to $S$ exactly $k-1$ times before reaching $b$ again. Due to propositions 4 and 5 , we can bound the expectation as follows: $E[V(S, b, b)]=\sum_{k=1}^{\infty} \tilde{q} k q^{k}(1-q)=\tilde{q} E[Z]$, where $Z$ is a geometric law of parameter $q$. Thus $E[Z]=\frac{1}{1-q}$ and $E[V(S, b, b)]=\frac{\tilde{q}}{1-q}=O\left(\frac{\epsilon l^{2}}{\epsilon}\right)$. The number of elements in the set $S$ is at most $l$, because we can index states in $\Omega_{A C}^{l}$ by the location of the three players who are at the same location. Therefore if $\operatorname{card}(B) \leq \operatorname{card}(S) \leq l$, $\lim _{N \rightarrow \infty} \frac{\mu^{N}(S)}{\mu^{N}(B)}=\lim _{N \rightarrow \infty} \frac{\mu^{N}(S)}{\sum_{i=1}^{i=c a r d(B)} \mu^{N}(b)}=\lim _{N \rightarrow \infty} 2 \operatorname{ll}[V(S, b, b)]=\infty$.

The lengthy proofs of Propositions 4 and 5, which are based on a number of Lemmas, have been placed in Appendices C.4 and C.5.

\subsection{Extension to more than four players}

In this section, we have provided a proof for the case $n=4$ players. For cases $n>4$, the dynamics starting from the center are similar: we move away from the center as long as all players do not locate on the same side. With probability $\left(\frac{1}{2}\right)^{n-1}$ all players end up on the same side and we return to the center. It would seem possible 
to generalize the proof regarding the probability of returning to the center to the case $n>4$. However, proving that a single random relocation takes us back from any location to the center becomes less manageable, because there is a large number of cases to consider. We conjecture that the behavior of the system for $n>4$ is similar to the case $n=4$, as is suggested by the numerical analysis.

Analyzing the dynamics also allows us to understand why the average distance to the center is larger when we increase the number of players. Indeed, the probability that all players end up on the same side and then return to the center is $p_{n}=:\left(\frac{1}{2}\right)^{n-1}$ at each time step, and thus the expected value of the first time that this occurs is $1 / p_{n}$

\subsection{Stochastic stability of Nash equilibria}

While we are not able to show, in the standard setting for equilibrium selection (for given $n \geq 4$ and with noise driven down to zero) that the stochastically stable steady states concentrate around the center, in this subsection we show that the pure strategy Nash equilibria of the model cannot be part of any of the stochastically stable steady states for any $n \geq 4$.

Theorem 3 Suppose that there are $n \geq 4$ players and that the number of locations $N$ is fixed and satisfies $N>n^{25}$ Then, no pure strategy Nash equilibrium is included in the set of stochastically stable steady states as the noise goes to zero.

We show that in a model without random noise, starting from any Nash equilibrium, there is a positive probability of reaching an absorbing state in $\Omega_{A C}$, implying that the Nash equilibrium cannot belong to another stochastically stable steady state. The key to proving the result is that the Nash equilibria are weak.

\footnotetext{
${ }^{25}$ Note that the condition $N>>n$ is required only to ensure that the number of locations between the players' distinct equilibrium positions will not be too small. If $n=4$, we also assume that $N$ is not a multiple of 4 .
} 
players are indifferent between their location in the Nash equilibrium and several other locations. Therefore, with positive probability they can move in such a way that a fairly large interval in the market is left unoccupied. In the next step, all players will prefer to locate within this interval and they will all end up on the same side of the center.

The proof is given in Appendix D. The analysis shows that when $n \geq 4$, the pure strategy Nash equilibria of the Hotelling modeling cannot be selected under 'trembling hand' dynamics. While we do not have a proof for this, we conjecture that the stochastically stable steady states will be the elements of $\Omega_{A C}$. However, some elements in $\Omega_{A C}$ are close to the center, others are not. While we expect the invariant measure to place more weight on the elements in $\Omega_{A C}$ that are closer to the center when the number of locations is large, the distinction becomes sharp only when we look at the asymptotic behavior with respect to the number of locations, as we showed earlier in Section 5.

\section{Welfare analysis}

In this section we analyze the welfare consequences of the best response dynamics relative to the Nash equilibrium from the point of view of voters for $n \geq 3$. Here we take the average distance of voters from the player located closest to them, relative to the total number of locations, as a measure of a welfare. The larger the average distance, the more electoral unhappiness, and the lower the welfare of the voters.

In the case of $n=3$, in the mixed strategy Nash equilibrium the lower bound for the average distance for the voters from the closest player is $3 / 32$, which happens when the three players are located equally distanced on $1 / 4,1 / 2$, and $3 / 4$ of the interval $[0,1]$. In the case of $n>3$, as noted in Section 2, in any pure strategy Nash equilibrium, players are located symmetrically in two sides of the center and are relatively uniformly spread across the interval, depending on the number of players 
(see Section 2 for details). The upper bound for any interval between players is $2 / n$, whereas the upper bound for the spaces beyond the peripheral players is $1 / n$. This means that the average distance of voters from the closest player is (at most) $1 /(2 n)$. Thus, for $n=4$ the average distance will be equal to $1 / 8$, while the average distance will get smaller and smaller as $n$ increases beyond 4 .

We can compare these average distances for the voters from the closest player in the Nash equilibria with the corresponding distances under the best response dynamics. Recall that, under the best response dynamics, as we observed in Figure 1, in case of $n=3$, the three players are essentially clustered in the center all the time. Thus, the average distance for voters from the closest player is $1 / 4$ of the total number of locations.

In case of $n>3$, the best response dynamics generate waves of outward expansions of two clusters of players equally distanced from the center and inward contractions of one clusters of all players who are all on the same side of the center. Let us denote $d \in[0,1 / 2]$ as the distance from the center relative to the total number of locations. When the players are split into two clusters of players that are symmetrically located on both sides of the center with some distance $d$ from the center, the average distance of the voters from the closest player is $\left(\frac{1}{2}-d\right)^{2}+d^{2}$. As we noted in Section 2, the upper bound for the outward waves is $d=1 / 4$. Thus, in this type of situation with two clusters, the average distance for the voters varies from $1 / 8($ when $d=1 / 4)$ to $1 / 4$ (when $d=0$ ). When, instead, all the players are clustered in the same location that is some distance $d$ from the center, the average distance for the voters is $\left(\frac{1}{2}-d\right)^{2} / 2+\left(\frac{1}{2}+d\right)^{2} / 2$. This distance varies between $1 / 4$ (when all the players are in the center, i.e. $d=0$ ) and 5/16 (when all the players are at $d=1 / 4$, the upper bound for the outward waves).

Thus, at any moment during the best response dynamics the theoretical lower bound for the average distance for the voters is $1 / 8$. Averaging over time means that 
the average distance for the best response dynamics will be strictly greater than $1 / 8$. What is more, we showed in Section 5 that when we let the number of locations $N$ go to infinity, under best response dynamics we see the players being located arbitrarily close to the center (where $d=0$ ), implying that the average distance for the voters will be $1 / 4$.

In other words, from a welfare point of view this means that the voters are strictly worse off under the best response dynamics than in any Nash equilibrium, and this is true for any $n \geq 3$.

\section{Concluding remarks}

We considered a linear location model (Hotelling, 1929) in which players follow noisy myopic best-replies. We asked what the likely configurations are in terms of numbers of players in each location in such a case.

We analyzed numerically how the average distance from the center depends on the number of players $n$ and the number of locations $2 M+1$, showing that by refining the discrete strategy space we can get the players locate arbitrarily close to the center almost all the time.

In our formal, mathematical analysis we proved, in the case of $n=3$ players, that all the players are located in close proximity of the center in the stochastically stable steady states. For the case of $n=4$ we proved that the players will tend to be located near the center if the noise is small (in a sense made precise) relative to the number of locations. The logic of the proof for $n=4$ appears applicable to any $n>4$ as well.

Although our analyses show that we do not necessarily always have all players located precisely in the center, and thus we do not always have the minimum differentiation as the Principle of Minimum Differentiation would suggest, our analyses suggest that, in contrast to the Nash equilibrium configuration for any 
$n \geq 3$, if players are myopic and adaptive we may tend to observe outcomes that conform rather closely to the Principle of Minimum Differentiation, with the difference becoming negligible as the space is refined and approximates a continuum. Thus, we restored Hotelling's principle for this class of boundedly rational players, and provided a possible explanation for the relatively common perception that decision makers in a wide range of situations tend to cater to the median voter even when there are more than two players: The return of the median voter.

As mentioned already in Section 1, and emphasized by Hotelling (1929) to start with, interpreting the location model as one of electoral competition is only one of the many possibilities, and the Principle of Minimum Differentiation applies to a wide range of situations of players competing in either discrete or continuous strategy spaces. Thus, our analysis of the best-response dynamics seems relevant in particular also for market dynamics, with the firms competing e.g. in geographical space or product characteristics space. ${ }^{26}$

Focussing on geographical space, our welfare analysis of Section 6 would apply to the total travel distance between the locations chosen by the firms and the preferred locations of the consumers. As Eaton and Lipsey (1975) already indicated, one important aspect of the multiplicity of the Nash equilibria in the Hotelling model is that these travel costs may differ from equilibrium to equilibrium. Our analysis adds a new dimension to this, as the best-response dynamics lead to outcomes that stand in stark contrast to all these Nash equilibria. It is not just that we do not get perfect convergence to the Nash equilibria. What we see is that these equilibria are not even approximated, as the system moves into other directions, with minimum differentiation quickly emerging. As our analysis shows, this implies an important welfare loss as less differentiation means substantially increased travel costs compared with any of the Nash equilibria.

\footnotetext{
${ }^{26}$ Firms may compete in other dimensions too. For example, Ewerhart (2014) discusses competition between professional forecasters with reputational concerns.
} 
Thus, to the extent that players may be inclined to adopt behavior resembling myopic best-replies, our analysis suggests that from a policy (welfare) point of view it may be important to look beyond an equilibrium analysis of such models. 


\section{References}

Balkenborg, D., J. Hofbauer, and C. Kuzmics (2013): "Refined best reply correspondence and dynamics," Theoretical Economics, 8, 165-192.

BlACK, D. (1948): "On the rationale of group decision-making," Journal of Political Economy, 5(1), 23-34.

Brown-Kruse, J., M. B. Cronshaw, and D. J. Schenk (1993): "Theory and experiments on spatial competition," Economic Inquiry, 31(1), 139-165.

Brown-Kruse, J., And D. J. Schenk (2000): "Location, Cooperation, and Communication: an experimental examination," International Journal of Industrial Organization, 18, 59-80.

Collins, R., And K. Sherstyuk (2000): "Spatial competition with three firms: an experimental study," Economic Inquiry, 38 (1), 73-94.

D’Aspremont, C., J. J. Gabszewicz, and J.-F. Thisse (1979): “On Hotelling's "Stability in Competition"," Econometrica, 47(5), 1145-1150.

Downs, A. (1957): An Economic Theory of Democracy. Harper Collins, New York.

Drouvelis, M., A. Saporiti, And N. J. Vriend (2014): "Political Motivations and Electoral Competition: Equilibrium Analysis and Experimental Evidence," Games and Economic Behavior, 83, 86-115.

Duverger, M. (1954): Political Parties. Methuen, London.

Eaton, B. C., And R. G. Lipsey (1975): "The Principle of Minimum Differentiation Reconsidered: Some New Developments in the Theory of Spatial Competition," Review of Economic Studies, 42(1), 27-49. 
Ellison, G. (2000): "Basins of Attraction, Long-Run Stochastic Stability, and the Speed of Step-by-Step Evolution," Review of Economic Studies, 67, 17-45.

Ewerhart, C. (2014): "Mixed Equilibrium in a Pure Location Game: The Case of $n \geq 4$ Firms," University of Zurich, Department of Economics, Working Paper No. 168.

Grofman, B. (2004): "Black's single peakedness condition," in The Encyclopedia of Public Choice, ed. by C. Rowley, and F. Schneider, pp. 43-45. Kluwer, Dordrecht/Boston/London.

Hofbauer, J. (1995): "Stability for the Best Response Dynamics," mimeo.

Hopkins, E. (1999): “A Note on Best Response Dynamics," Games and Economic Behavior, 29, 138-150.

Hotelling, H. (1929): "Stability in Competition," Economic Journal, 39(153), $41-57$.

Huck, S., W. Müller, And N. J. VRIEnd (2002): "The east end, the west end, and King's cross: On clustering in the four-player hotelling game," Economic Inquiry, 40(2), 231-240.

Irmen, A., AND J.-F. Thisse (1998): "Competition in Multi-Characteristics Spaces: Hotelling Was Almost Right," Journal of Economic Theory, 78, 76-102.

Kemeny, J. G., and J. L. Snell (1960): Finite Markov Chains. D. van Nostrand Co. Ltd., London.

Matsumura, T., N. Matsushima, and T. Yamanori (2010): "Evolution of competitive equilibrium with endogenous product differentiation," mimeo, Institute of Social and Economic Research, Osaka University, Osaka, Japan. 
Osborne, M. J. (1995): "Spatial Models of Political Competition under Plurality Rule: A Survey of Some Explanations of the Number of Candidates and the Positions They Take," Canadian Journal of Economics/Revue canadienne d'Economique, 28(2), 261-301.

Palma, A. D., V. Ginsburgh, Y. Y. Papageorgiou, and J.-F. Thisse (1985): "The Principle of Minimum Differentiation Holds under Sufficient Heterogeneity," Econometrica, 53(4), 767-781.

PAnCs, R., And N. J. VRIEnd (2007): "Schelling's spatial proximity model of segregation revisited," Journal of Public Economics, 91, 1-24.

Riker, W. H. (1982): “The Two-Party System and Duverger's Law: An Essay on the History of Political Science," American Political Science Review, 76(4), 753-766.

Rowley, C. K. (1984): "The Relevance of the Median Voter Theorem," Zeitschrift für die gesamte Staatswissenschaft/Journal of Institutional and Theoretical Economics, 140(1), 104-126.

SHAKED, A. (1982): "Existence and computation of mixed strategy Nash Equilibrium for 3-firms location problem," Journal of Industrial Economics, $31(1 / 2), 93-96$.

Shino, J., And R. KaWASAKI (2012): "Farsighted stable sets in Hotelling's location games," Mathematical Social Sciences, 63, 23-30. 


\section{A Appendix: Robustness checks for numerical analysis}

In this appendix we report the numerical analysis for variants of the basic setup in which we consider (1) an even number of locations, (2) other values for the probability of random moves, (3) non-simultaneous moves, and (4) inertia in the location choice when players cannot find a strictly better location.

\section{A.1 Even number of locations}

When there is an even number of locations, there is no possibility for the system to converge to the center in the sense that the median is right in-between the two central locations. For example, with $N=3002$ locations (indexed by $\{0,1,2, \ldots, 3001\}$, the median $M$ would be at 1500.5 , which is not a location that can be chosen by the players. This is why we focused our analysis in the main text on the case of odd numbers of locations. But it may nevertheless be interesting to see how the dynamics may differ in the case of even numbers of locations. Table 2 reports the mean distances from the center (with standard deviations in parentheses) with some even numbers of locations. ${ }^{27}$ Compared to the case with the odd numbers of locations reported in Table 1, the mean distance is substantially smaller, especially for larger $n$.

As is illustrated for the case of $M=1500.5$ in Figure 2, this is related to some qualitative differences in the best-response dynamics. Note that there is a difference in speed of the outward movements in Figure 1 (with an odd number of locations) and Figure 2 (with an even number of locations), this being lower than in the case of an odd number of of locations. Recall from our main analysis that in the case of an odd number of locations the players would repeatedly approach the median $M$

\footnotetext{
${ }^{27}$ To compute these distances to the center we treat the two nearest locations as having a distance of 0 .
} 


\begin{tabular}{ccccccc}
\hline \multicolumn{7}{c}{$M=1500.5$} \\
\hline & $\mathrm{n}=3$ & $\mathrm{n}=4$ & $\mathrm{n}=5$ & $\mathrm{n}=6$ & $\mathrm{n}=7$ & $\mathrm{n}=8$ \\
\hline $90 \%$ & 2.0 & 4.0 & 5.1 & 7.0 & 8.0 & 9.0 \\
& $(0.0)$ & $(0.0)$ & $(0.3)$ & $(0.0)$ & $(0.0)$ & $(0.0)$ \\
$95 \%$ & 2.0 & 6.0 & 7.0 & 9.0 & 10.0 & 12.0 \\
& $(0.0)$ & $(0.0)$ & $(0.0)$ & $(0.0)$ & $(0.0)$ & $(0.2)$ \\
$99 \%$ & 2.0 & 10.0 & 12.1 & 15.0 & 18.4 & 22.0 \\
& $(0.0)$ & $(0.0)$ & $(0.3)$ & $(0.2)$ & $(0.5)$ & $(0.2)$ \\
\hline
\end{tabular}

\begin{tabular}{ccccccc}
\multicolumn{7}{c}{$M=15000.5$} \\
\hline & $\mathrm{n}=3$ & $\mathrm{n}=4$ & $\mathrm{n}=5$ & $\mathrm{n}=6$ & $\mathrm{n}=7$ & $\mathrm{n}=8$ \\
\hline $90 \%$ & 2.0 & 4.0 & 6.0 & 7.0 & 8.0 & 9.0 \\
& $(0.0)$ & $(0.0)$ & $(0.3)$ & $(0.0)$ & $(0.0)$ & $(0.0)$ \\
$95 \%$ & 2.0 & 6.0 & 7.0 & 9.0 & 10.4 & 12.0 \\
& $(0.0)$ & $(0.0)$ & $(0.2)$ & $(0.0)$ & $(0.5)$ & $(0.0)$ \\
$99 \%$ & 2.0 & 10.0 & 12.9 & 16.0 & 19.5 & 24.0 \\
& $(0.0)$ & $(0.0)$ & $(0.4)$ & $(0.0)$ & $(0.5)$ & $(0.6)$ \\
\hline
\end{tabular}

\begin{tabular}{ccccccc}
\multicolumn{7}{c}{$M=150000.5$} \\
\hline & $\mathrm{n}=3$ & $\mathrm{n}=4$ & $\mathrm{n}=5$ & $\mathrm{n}=6$ & $\mathrm{n}=7$ & $\mathrm{n}=8$ \\
\hline $90 \%$ & 2.0 & 4.0 & 6.0 & 7.0 & 8.0 & 9.0 \\
& $(0.0)$ & $(0.0)$ & $(0.0)$ & $(0.0)$ & $(0.0)$ & $(0.0)$ \\
$95 \%$ & 2.0 & 6.0 & 7.0 & 9.0 & 10.6 & 12.0 \\
& $(0.0)$ & $(0.0)$ & $(0.2)$ & $(0.0)$ & $(0.5)$ & $(0.0)$ \\
$99 \%$ & 2.0 & 10.0 & 13.0 & 16.0 & 19.7 & 24.1 \\
& $(0.0)$ & $(0.0)$ & $(0.0)$ & $(0.0)$ & $(0.5)$ & $(0.6)$ \\
\hline
\end{tabular}

Table 2: Mean distances from the center (with standard deviations in parentheses) with even numbers of locations, $n=3,4,5,6,7,8$, with $\epsilon=0.001$

together. Once there, they faced two equally attractive locations, one immediately to the left and one immediately to the right. The players would, then, be likely to split into two smaller groups, starting their steadily outward movement, one location for each time-step. The outward movement continues until the two-group configuration is shattered, which typically happens randomly as each player chooses randomly between the two sides. With an even number of locations, however, there is no such steadily outward movement. There is no median location available, and if all players are located immediately to the left of the median, then the unique 
$n=3$
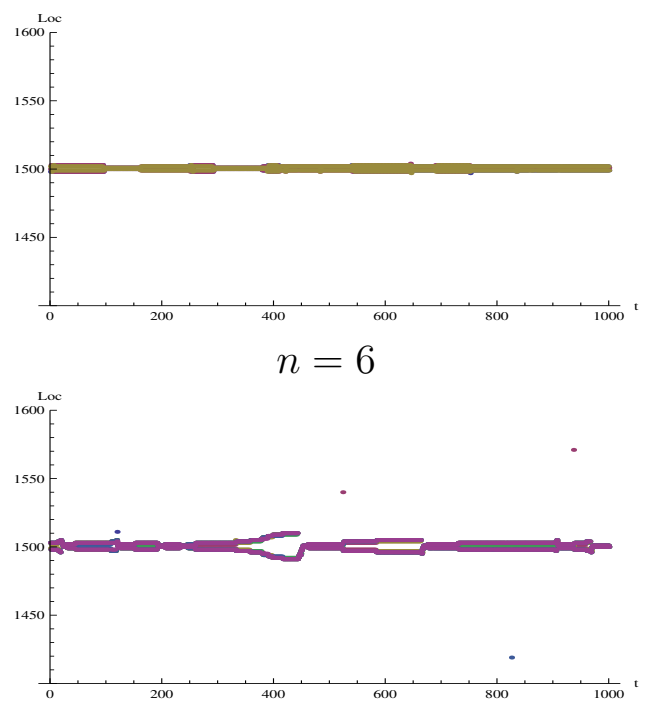

$n=4$
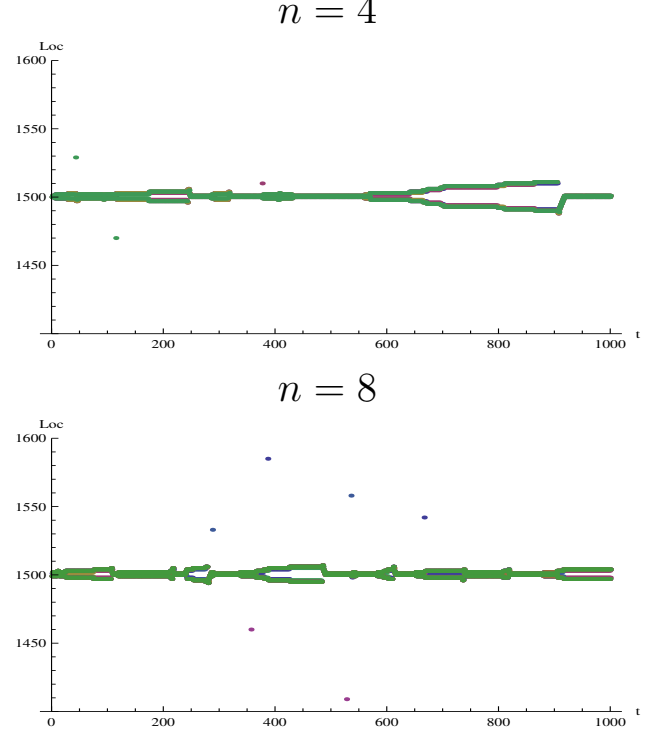

Figure 2: Locations over 1000 period interval for $M=1500.5, n=3,4,6,8$, with $\epsilon=0.001$

best-response is the location immediately to the right of the median, and the other way around. Alternatively, the players may find themselves in an absorbing class similar to those at the end of Proposition 1, with two groups of players located approximately symmetrically around the center. In either case their distance to the center is constant. The slow outward movement that we observe with an even number of locations is due to individual random moves plus subsequent best-replies, typically resulting in some small one-off outward movement.

\section{A.2 Other levels of noise in the myopic best reply}

In our main analysis we considered the probability of random choice to be $\epsilon=0.001$. In this appendix, we consider $\epsilon \in\{0.01,0.001,0.0001,0.00001,0.00000\}$ to see the limiting results as we reduce the error rate. We concentrate here on odd numbers of locations and simultaneous moves. Table 3 reports the average distance below which the system will spend $99 \%$ of the time. As the table clearly shows, with $\epsilon=0.01$, a much higher noise level than the $\epsilon=0.001$ used in our main analysis, the basic, 
dispersing effect of random choices starts to dominate and as a result the distance from the center would increase substantially. But considering lower noise levels than $\epsilon=0.001$ does not qualitatively change the picture of our main analysis.

\section{A.3 Non-simultaneous move}

In the main text we considered simultaneous, noisy best-responses, where all players decide their location simultaneously in each period. We now relax this assumption and consider the case where in each period, one randomly chosen player decides about his location. In order to keep number of decisions being made the same as with the simultaneous move case, we ran the model $n$ times as many time steps as in our main analysis with simultaneous moves, such that the expected number of decisions per player is the same in both variants. ${ }^{28}$

Table 4 shows the results for this variant. Compared to the simultaneous move case, the average distance is higher, especially for higher values of $M$. This is due to the following difference with our main analysis. With simultaneous moves, for any random mistake the players can adjust their location choices immediately. But without simultaneous moves this is different. A single random move can make the average distance from the center quite large, and it may take some time before the randomly re-located player is selected again to correct his choice. Thus, for any given noise level, the effect of the random mistakes is much more prolonged than with simultaneous moves. As we saw already in Table 3, for too high noise levels we get substantially greater distances from the center. To illustrate further that it is indeed the prolonged effect of the noise that causes these distances to increase with non-simultaneous moves, we computed for this variant the distances from the center below which the system will spend $99 \%$ of the time with a noise level of $\epsilon=0.0001$

\footnotetext{
${ }^{28}$ Thus, the analysis here is based on the $11 \times T$ periods with $T=10,000,000 \times n$, where $n$ is the number of players, and each player is still making, on average, $11 \times 10$ millions decisions. As before, we drop the data from the first $T$ periods and use the data for the remaining $10 \times T$ periods.
} 


\begin{tabular}{lcccccc}
\hline \multicolumn{7}{c}{$M=1500$} \\
\hline & $\mathrm{n}=3$ & $\mathrm{n}=4$ & $\mathrm{n}=5$ & $\mathrm{n}=6$ & $\mathrm{n}=7$ & $\mathrm{n}=8$ \\
\hline$\epsilon=0.01$ & 336.3 & 286.6 & 252.0 & 227.0 & 208.0 & 191.0 \\
& $(0.5)$ & $(0.5)$ & $(0.0)$ & $(0.2)$ & $(0.0)$ & $(0.0)$ \\
$\epsilon=0.001$ & 2.0 & 15.0 & 65.9 & 139.9 & 247.4 & 409.3 \\
& $(0.0)$ & $(0.0)$ & $(0.4)$ & $(0.3)$ & $(0.8)$ & $(1.4)$ \\
$\epsilon=0.0001$ & 2.0 & 14.0 & 61.0 & 138.9 & 283.7 & 522.3 \\
& $(0.0)$ & $(0.0)$ & $(0.0)$ & $(0.4)$ & $(1.0)$ & $(1.7)$ \\
$\epsilon=0.00001$ & 2.0 & 14.0 & 60.9 & 138.9 & 288.7 & 534.8 \\
& $(0.0)$ & $(0.0)$ & $(0.3)$ & $(0.4)$ & $(1.5)$ & $(2.3)$ \\
$\epsilon=0.00000$ & 2.0 & 14.0 & 60.9 & 138.9 & 288.9 & 495.2 \\
& $(0.0)$ & $(0.0)$ & $(0.3)$ & $(0.3)$ & $(1.0)$ & $(82.0)$ \\
\hline
\end{tabular}

\begin{tabular}{lcccccc}
\hline \multicolumn{7}{c}{$M=15000$} \\
\hline$\epsilon=0.01$ & $\mathrm{n}=3$ & $\mathrm{n}=4$ & $\mathrm{n}=5$ & $\mathrm{n}=6$ & $\mathrm{n}=7$ & $\mathrm{n}=8$ \\
\hline \multirow{4}{*}{$=0.001$} & 3351.8 & 2838.1 & 2445.6 & 2144.6 & 1909.8 & 1721.5 \\
& $2.7)$ & $(1.1)$ & $(1.2)$ & $(0.9)$ & $(0.8)$ & $(0.7)$ \\
$\epsilon=0.0001$ & 2.0 & 15.0 & 67.2 & 152.0 & 291.5 & 481.9 \\
& $(0.0)$ & $(0.0)$ & $(0.4)$ & $(0.5)$ & $(1.5)$ & $(2.4)$ \\
$\epsilon=0.00001$ & 2.0 & 14.0 & 61.0 & 139.7 & 287.2 & 567.5 \\
& $(0.0)$ & $(0.0)$ & $(0.0)$ & $(0.5)$ & $(1.1)$ & $(2.6)$ \\
$\epsilon=0.00000$ & 14.0 & 60.9 & 139.0 & 288.9 & 583.5 \\
& $(0.0)$ & $(0.0)$ & $(0.3)$ & $(0.4)$ & $(1.0)$ & $(2.8)$ \\
& 2.0 & 14.0 & 60.9 & 138.9 & 288.9 & 585.8 \\
& $(0.0)$ & $(0.0)$ & $(0.3)$ & $(0.3)$ & $(1.0)$ & $(3.0)$ \\
\hline
\end{tabular}

\begin{tabular}{lcccccc}
\hline \multicolumn{7}{c}{$M=15000$} \\
\hline$\epsilon=0.01$ & $\mathrm{n}=3$ & $\mathrm{n}=4$ & $\mathrm{n}=5$ & $\mathrm{n}=6$ & $\mathrm{n}=7$ & $\mathrm{n}=8$ \\
\hline \multirow{4}{*}{$=0.001$} & 33514.1 & 28354.9 & 24382.7 & 21323.0 & 18942.9 & 17054.1 \\
& $25.7)$ & $(10.7)$ & $(12.2)$ & $(8.1)$ & $(7.1)$ & $(7.9)$ \\
$\epsilon=0.0001$ & 2.0 & 15.0 & 67.9 & 154.0 & 302.0 & 525.6 \\
& 2.0 & $(0.0)$ & $(0.3)$ & $(0.5)$ & $(1.4)$ & $(3.8)$ \\
$\epsilon=0.00001$ & 2.0 & 14.0 & 61.0 & 139.7 & 287.7 & 569.3 \\
& $(0.0)$ & $(0.0)$ & $(0.0)$ & $(0.5)$ & $(1.1)$ & $(2.9)$ \\
$\epsilon=0.00000$ & 14.0 & 60.9 & 139.0 & 289.0 & 583.8 \\
& 2.0 & 14.0 & 60.9 & 138.9 & 288.9 & 585.8 \\
& $(0.0)$ & $(0.0)$ & $(0.3)$ & $(0.3)$ & $(1.0)$ & $(3.1)$ \\
\hline
\end{tabular}

Table 3: Mean distances from the center for $99 \%$ of time (with standard deviations in parentheses) for various $\epsilon, M=1500,15,000,150,000, n=3,4,5,6,7,8$ 
instead of $\epsilon=0.001$. This reduces the average distances drastically. ${ }^{29}$

\section{A.4 Presence of inertia}

The reason for the instability of Nash equilibria was that they are weak in the sense that players were indifferent between the equilibrium locations and those located between equilibrium locations as long as others remain in their equilibrium locations. Therefore, we consider the role of inertia. That is, we analyze the dynamics if players simply choose to stay put in case there is no location that is strictly better than their current location. Otherwise the behavior is the same as in our main analysis, i.e., the players choose a best-reply and choose randomly among their best-replies if there is more than one.

Table 5 shows the results for an odd number of locations and simultaneous moves, which we can compare with Table 1 . As we can see, inertia does not change the picture, and thus the lack of inertia in our main analysis was not crucial for our results. This is due to the fact that the dynamics will normally never reach any of the Nash equilibria.

\section{B Appendix: Proofs for the three player case}

\section{B.1 Lemma 2}

Proof: Because we have shown that when all the three players are on the same side ( $A O S$, for 'all one side'), we are in $B(S)$, we consider the remaining cases.

There are 19 cases that we need to consider (up to symmetry). And, for each case, we will show the best response path either reaches the state where all the players are on the same side $A O S$ or $S$.

\footnotetext{
${ }^{29}$ For $n$ equal to $3,4,5,6,7$ and 8 these distance would become 4.0, 15.3, 60.1, 241.4, 976.3 and 3582.8 respectively, for $\mathrm{M}=150,000$.
} 


\begin{tabular}{ccccccc}
\hline \multicolumn{7}{c}{$M=1500$} \\
\hline & $\mathrm{n}=3$ & $\mathrm{n}=4$ & $\mathrm{n}=5$ & $\mathrm{n}=6$ & $\mathrm{n}=7$ & $\mathrm{n}=8$ \\
\hline $90 \%$ & 3.0 & 9.0 & 31.0 & 116.6 & 394.1 & 670.0 \\
& $(0.0)$ & $(0.0)$ & $(0.0)$ & $(0.5)$ & $(0.6)$ & $(0.2)$ \\
$95 \%$ & 4.0 & 11.0 & 40.0 & 152.0 & 491.2 & 726.0 \\
& $(0.0)$ & $(0.0)$ & $(0.0)$ & $(0.0)$ & $(0.8)$ & $(0.0)$ \\
$99 \%$ & 5.0 & 17.0 & 66.0 & 235.4 & 676.4 & 777.1 \\
& $(0.0)$ & $(0.0)$ & $(0.2)$ & $(0.5)$ & $(0.6)$ & $(0.3)$ \\
\hline
\end{tabular}

\begin{tabular}{ccccccc}
\multicolumn{7}{c}{$M=15,000$} \\
\hline & $\mathrm{n}=3$ & $\mathrm{n}=4$ & $\mathrm{n}=5$ & $\mathrm{n}=6$ & $\mathrm{n}=7$ & $\mathrm{n}=8$ \\
\hline $90 \%$ & 3.0 & 9.0 & 31.0 & 118.0 & 426.2 & 1280.3 \\
& $(0.0)$ & $(0.0)$ & $(0.0)$ & $(0.0)$ & $(1.0)$ & $(3.8)$ \\
$95 \%$ & 4.0 & 11.0 & 40.6 & 155.3 & 563.3 & 1677.6 \\
& $(0.0)$ & $(0.0)$ & $(0.5)$ & $(0.5)$ & $(1.2)$ & $(5.5)$ \\
$99 \%$ & 5.0 & 18.0 & 70.9 & 281.8 & 968.1 & 2607.0 \\
& $(0.0)$ & $(0.0)$ & $(0.5)$ & $(1.5)$ & $(3.8)$ & $(11.6)$ \\
\hline
\end{tabular}

\begin{tabular}{ccccccc}
\multicolumn{7}{c}{$M=150,000$} \\
\hline & $\mathrm{n}=3$ & $\mathrm{n}=4$ & $\mathrm{n}=5$ & $\mathrm{n}=6$ & $\mathrm{n}=7$ & $\mathrm{n}=8$ \\
\hline $90 \%$ & 3.0 & 9.0 & 32.0 & 120.7 & 438.2 & 1306.1 \\
& $(0.0)$ & $(0.0)$ & $(0.0)$ & $(0.5)$ & $(1.3)$ & $(4.3)$ \\
$95 \%$ & 4.0 & 11.8 & 42.1 & 162.6 & 594.5 & 1775.2 \\
& $(0.0)$ & $(0.4)$ & $(0.3)$ & $(0.7)$ & $(2.5)$ & $(8.1)$ \\
$99 \%$ & 8119.8 & 622.5 & 2953.5 & 4922.6 & 6380.7 & 7482.5 \\
& $(1629.7)$ & $(545.7)$ & $(865.6)$ & $(650.0)$ & $(533.1)$ & $(499.0)$ \\
\hline
\end{tabular}

Table 4: Mean distances from the center (with standard deviations in parentheses) when one randomly chosen player moving at a time for $M=1500,15,000,150,000$, $n=3,4,5,6,7,8 . \epsilon=0.001$ 


\begin{tabular}{ccccccc}
\hline \multicolumn{7}{c}{$M=1500$} \\
\hline & $\mathrm{n}=3$ & $\mathrm{n}=4$ & $\mathrm{n}=5$ & $\mathrm{n}=6$ & $\mathrm{n}=7$ & $\mathrm{n}=8$ \\
\hline $90 \%$ & 2.0 & 8.0 & 30.0 & 66.0 & 123.7 & 203.8 \\
& $(0.0)$ & $(0.0)$ & $(0.0)$ & $(0.0)$ & $(0.5)$ & $(0.7)$ \\
$95 \%$ & 2.0 & 10.0 & 40.0 & 86.9 & 161.3 & 265.5 \\
& $(0.0)$ & $(0.0)$ & $(0.0)$ & $(0.3)$ & $(0.5)$ & $(0.9)$ \\
$99 \%$ & 2.0 & 15.0 & 65.9 & 139.9 & 247.5 & 409.6 \\
& $(0.0)$ & $(0.0)$ & $(0.45)$ & $(0.3)$ & $(0.8)$ & $(1.4)$ \\
\hline
\end{tabular}

\begin{tabular}{ccccccc}
\multicolumn{7}{c}{$M=15000$} \\
\hline & $\mathrm{n}=3$ & $\mathrm{n}=4$ & $\mathrm{n}=5$ & $\mathrm{n}=6$ & $\mathrm{n}=7$ & $\mathrm{n}=8$ \\
\hline $90 \%$ & 2.0 & 8.0 & 30.0 & 66.1 & 124.1 & 204.6 \\
& $(0.0)$ & $(0.0)$ & $(0.0)$ & $(0.3)$ & $(0.4)$ & $(0.5)$ \\
$95 \%$ & 2.0 & 10.0 & 40.0 & 87.3 & 164.3 & 271.1 \\
& $(0.0)$ & $(0.0)$ & $(0.0)$ & $(0.5)$ & $(0.5)$ & $(0.9)$ \\
$99 \%$ & 2.0 & 15.0 & 67.2 & 152.0 & 291.5 & 471.7 \\
& $(0.0)$ & $(0.0)$ & $(0.4)$ & $(0.5)$ & $(1.5)$ & $(2.3)$ \\
\hline
\end{tabular}

\begin{tabular}{ccccccc}
\multicolumn{7}{c}{$M=150000$} \\
\hline & $\mathrm{n}=3$ & $\mathrm{n}=4$ & $\mathrm{n}=5$ & $\mathrm{n}=6$ & $\mathrm{n}=7$ & $\mathrm{n}=8$ \\
\hline $90 \%$ & 2.0 & 8.0 & 30.0 & 66.1 & 124.3 & 204.7 \\
& $(0.0)$ & $(0.0)$ & $(0.0)$ & $(0.4)$ & $(0.5)$ & $(0.5)$ \\
$95 \%$ & 2.0 & 10.0 & 40.0 & 87.5 & 164.6 & 272.0 \\
& $(0.0)$ & $(0.0)$ & $(0.0)$ & $(0.5)$ & $(0.5)$ & $(0.8)$ \\
$99 \%$ & 2.0 & 15.0 & 67.9 & 154.0 & 302.2 & 526.9 \\
& $(0.0)$ & $(0.0)$ & $(0.3)$ & $(0.5)$ & $(1.7)$ & $(3.3)$ \\
\hline
\end{tabular}

Table 5: Mean distances from the center (with standard deviations in parentheses) in presence of intertia for $M=1500,15,000,150,000 . n=3,4,5,6,7,8$, with $\epsilon=0.001$

(i) $[M-3, M-3, M+3] \rightarrow$ either

- $[M-4, M-4, M-2] \in A O S$

- $[M+4, M+4, M-2] \rightarrow[M-1, M-1, M+3]$, which will be considered below (xi)

- $[M-4, M+4, M-2] \rightarrow$ either

$$
\begin{aligned}
& -[M-5, M-1, M-5] \in A O S \\
& -[M-3, M-1, M+5] \rightarrow[M-2, M-4, M] \in A O S
\end{aligned}
$$


(ii) $[M-3, M-2, M+3] \rightarrow$ either

- $[M-3, M-4, M-1] \in A O S$

- $[M-3, M+4, M-1] \rightarrow[M-2, M, M-4] \in A O S$

(iii) $[M-3, M-1, M+3] \rightarrow$ either

- $[M-2, M-4, M] \in A O S$

- $[M-2, M+4, M] \rightarrow[M-1, M-3, M+1] \in S$

(iv) $[M-3, M, M+3] \rightarrow$ either

- $[M-1, M+4, M+1] \rightarrow$ either $[M, M+2, M-2] \in S$ or $[M+2, M+2, M] \in$ $S$

- $[M-1, M-4, M+1] \rightarrow$ either $[M+2, M+2, M] \in S$ or $[M+2, M-2, M] \in$ $S$

(v) $[M-3, M-3, M+1] \rightarrow[M+2, M+2, M-2]$ to be considered below (xiv)

(vi) $[M-3, M-2, M+1] \rightarrow[M+2, M+2, M-1] \in S$

(vii) $[M-3, M-2, M+2] \rightarrow[M+3, M+3, M-1]$ considered above (v)

(viii) $[M-3, M-1, M+2] \rightarrow[M+3, M+3, M] \in A O S$

(ix) $[M-3, M, M+1] \rightarrow[M+2, M+2, M+1] \in A O S$

(x) $[M-3, M, M+2] \rightarrow[M+3, M+3, M+1] \in A O S$

(xi) $[M-3, M+1, M+1] \rightarrow[M, M-2, M-2] \in S$

(xii) $[M-3, M+1, M+2] \rightarrow[M, M+3, M+2] \in A O S$

(xiii) $[M-3, M+2, M+2] \rightarrow[M+1, M-2, M-2] \in S$

(xiv) $[M-2, M-2, M+2] \rightarrow$ either 
- $[M-3, M-3, M-1] \in A O S$

- $[M-3, M+3, M-1]$ considered above (iii)

(xv) $[M-2, M-1, M+1] \rightarrow$ either

- $[M-2, M+2, M] \in S$

- $[M+2, M+2, M] \in S$

(xvi) $[M-2, M-1, M+2] \rightarrow$ either

- $[M-2, M+3, M]$ considered above (x)

- $[M-2, M-3, M] \in A O S$

(xvii) $[M-2, M, M+1] \rightarrow$ either

- $[M-1, M+2, M+1]$ considered above (xv)

- $[M, M+2, M-2] \in S$

(xviii) $[M-2, M+1, M+1] \rightarrow[M-2, M-2, M] \in S$

(xix) $[M-1, M, M+1] \rightarrow$ either

- $[M-1, M-2, M+1]$ considered above (xv)

- $[M-1, M+2, M+1]$ considered above (xv)

\section{B.2 Lemma 3}

Proof: Let $s=(a, b, c) \in S$, and consider all possible cases. 
- First case, we relocate the middle player $b$ : If he is relocated in $[0, M-4]$, then $B R^{r}=a+1, B R^{m}=c+1$ and the best reply of the left player is $a-1$ or $c+1$. We are in $\{\omega \mid \omega \in[M-3, M+3]\}-C_{3}$ unless $c=M+3$ or $a=M-3$. In the first case $s=(M-1, M+1, M+3)$ and $B R=(M-2, M, M+4)$ but then $B R_{2}=(M-3, M-1, M+1) \in S$. In the second case $s=(M-3, M-1, M+1)$ and $B R_{2}=(M-2, M, M+2) \in S$. The case where he is relocated on the right is identical by symmetry.

- Second, we relocate the rightmost player $c$ : If we relocate him on the left we are in $[0, M]$. If he is relocated on $[M+4,2 M]$, then $B R=(a-1, b-1, b+1)$. This is in $[M-3, M+3]-C_{3}$ unless $a=M-3$. But then $s=(M-3, M-1, M+1)$ and $B R=(M-4, M-2, M) \in[0, M]$.

- Third, we relocate the leftmost player $a$ : If he is relocated in $[0, M-4]$, then $B R^{r}=b+1, B R^{m}=c+1$ and $B R^{l}$ is either $b-1$ or $c+1$. We are in $\{\omega \mid \omega \in[M-3, M+3]\}-C_{3}$ unless $c=M+3$. If this is the case $s=(M-1, M+1, M+3)$ and $B R=(M, M+2, M+4) \in[M, 2 M]$. If we relocate the leftmost player at $a$ in $[M+4,2 M]$, then $B R^{l}=c-1, B R^{m}=b-1$ and $B R^{r}$ is either $c+1$ or $b-1$. We are in $[M-3, M+3]-C_{3}$ unless $c=M+3$ but in this last case, $B R=(M+2, M, M) \in[M, 2 M]$.

- Finally, we need to check that we cannot get to $C_{3}$ by relocating a single player in a configuration $s \in S$. Indeed, we need to have two players at $M-3$ or at $M+3$. Consider $s=(M-1, M+1, M+3)$ and $(M-3, M-1, M+1)$. We cannot get from these to $C_{3}$ by moving a single player. 


\section{Appendix: Proofs for the four player case}

\section{C.1 Typical sequences: bounding the probability of rare events}

Scenarios where two or more random relocations occur close to each other are very unlikely. It will be useful to bound the probability of such an event to show that it is very small. This is the objective of Lemma 4. When we study the dynamics, we will then condition on the fact that there is at most one random relocation in a given number of periods.

Definition 1 We will say that a sequence is typical if it contains at most one random relocation and this relocation is not located in $I$.

Lemma 4 The probability that a sequence of length $l$ is not typical is at most $O\left(\frac{\epsilon l^{2}}{N}\right)$.

Proof: The probability of at least two random relocations in a sequence of length $l$ is $\sum_{k=2}^{k=4 l} \epsilon^{k}(1-\epsilon)^{4 l-k} C_{k}^{4 l} \leq \sum_{k=2}^{k=4 l} \epsilon^{k}(4 l)^{k}=\frac{(4 l \epsilon)^{2}-(4 l \epsilon)^{4 l+1}}{1-4 l \epsilon}=O\left(\epsilon^{2} l^{2}\right)$. Note that we have $4 l$ because at each date, there are four players who each relocate with probability $\epsilon$. The probability that there is exactly one random relocation and that it is in $I$ is smaller than $4 l \epsilon \frac{l}{N}=O\left(\frac{l^{2} \epsilon}{N}\right)$.

\section{C.2 Best reply dynamics starting from a state close to the center}

In what follows, a state will be $\omega=:(a, b, c, d)$ with $a \leq b \leq c \leq d$ and $l(\omega)=d-a$.

Lemma 5 tells us that starting from a configuration close to the center and assuming the sequence is typical, best replies will either take us closer to the center 
than before, or, if there is a positive probability of moving farther away from the center, there is also a positive probability that all the players end up on the same side, or, as a final possibility, we may enter an absorbing class close to the center.

Lemma 5 Assume that the evolution is 'typical' as in Definition 1 above. Any state $\omega \in I-S$ belongs to $\bigcup_{i=1}^{i=4} \Omega_{i}$ where:

- If $\omega \in \Omega_{1}$, Then $P\left(\left(B R_{4}(\omega) \cup B R_{5}(\omega)\right)=\bar{x} \in I\right) \geq p\left(p \geq \frac{1}{8^{3}}\right)$ and if this does not occur, $d\left(B R^{2}(\omega), M\right) \leq d(\omega, M)+2$.

- If $\omega \in \Omega_{2}$, then $d(B R(\omega), M) \leq d(\omega, M)$ and $l(B R(\omega)) \leq l(\omega)$, with at least one strict inequality.

- If $\omega \in \Omega_{3}$, then $B R(\omega)$ is at the same distance to the center as $\omega$ and $B R(\omega) \in \Omega_{1} \cup \Omega_{2}$.

- If $\omega \in \Omega_{4}$, then $B R_{2}(\omega)$ belongs to an absorbing class in $\Omega_{A C}^{l}$ with $l<$ $d(\omega, M)+2$.

Proof: (of Lemma 5) First, we prove that Lemma 5 holds at a date $t$ where no random perturbation occurs. Let us prove that any state $(a, b, c, d) \in I$ belongs to one of the aforementioned categories.

- First, consider $a<b \leq c<d$. Note that there are two possible cases, either there are two players on each side of the center, or three players on one side and one player alone on the other side. Sometimes, but not always, it is necessary to treat these cases separately.

- First, consider the case $d(a, M)=d(d, M)$. If $c<M$, the best reply $(a-1, a-1, b-1, c+1) \in A$ has positive probability, so we are in $\Omega_{1}$. Suppose from now on that $c \geq M$. If $d(b-1, M) \geq d(c+3, M)$, with positive probability $B R=(a-1, a-1, b-1, c+1), B R_{2}=(b,(c+2) * 3)$, 
and with positive probability $B R_{3}=(c+1,(c+3) * 3) \in A$. If $d(b-1, M)<$ $d(c+3, M)$, with positive probability $B R=(d+1, d+1, b-1, c+1)$, $B R_{2}=(c,(b-2) * 3)$, and $B R_{3}=(b-1,(b-3) * 3) \in A$.

- Suppose now without loss of generality that $d(d, M)<d(a, M)$, that is players prefer location $d+1$ to $a-1$.

* If $d(d+1, M)<d(a, M)$, then the maximal endpoint has contracted, we are in $\Omega_{2}$.

* If $d(d+1, M)=d(a, M)$, then if $d(b, M)>d(a+2, M), B R^{t}=$ $(b-1, d+1, d+1, c+1)$ and $l\left(B R_{t}(\omega)\right)=(d+1)-(b-1)<d-a=l(\omega)$. Thus, $\omega \in \Omega_{2}$.

- If $d(b, M)=d(a+2, M), B R_{t}=(a+1, c+1, d+1, d+1)$ and $B R_{t+1}=(a, a, a, c)$, which has contracted.

- If $d(b, M)=d(a+1, M)$, we can have $B R_{t}=(a, c+1, d+1, d+1)$, as $a$ is indifferent between staying put and $d+1$. In the next step, $a$ will relocate at $c$. All others are indifferent between $a-1$ and $d+2$, so with positive probability they end up on the same side as $c$ so that $A$ is reached. Thus we are in $\Omega_{1}$.

- Now consider the case $(a, b, c, c)$ with $a<b<c$, where there are two players at one of the endpoints.

- If $d(a, M)=d(c, M)$, we are in $\Omega_{1}$ because due to indifference, there is a positive probability that all players end up on the same side as $b-1$.

- If $d(a, M) \geq d(c+1, M), B R_{t}=((c+1) * 3, b-1)$. Thus, we assume $b \leq M$. Since $c+1-(b-1)=c-b+2$, the interval has contracted unless $b \in\{a+1, a+2\}$. If $d(b, M)=d(a+1, M), \omega=(a, a+1, c, c)$ and $(c+1) * 4 \in A \in B R_{t}$. If $b=a+2$, and if $d(a, M)>d(c+1, M)$, 
with positive probability $B R_{t}=(c+1) * 4 \in A$. If $d(a, M)=d(c+1, M)$, $B R_{t}=(a+1,(c+1) * 3)$ which is an absorbing state. We are then in $\Omega_{4}$. - If $d(a, M) \leq d(c-1, M), B R_{t}=((a-1) * 3, b-1)$, and since $b-1-(a-1)=b-a<c-a$, we are in $\Omega_{2}$.

- If the configuration is $(a, c, c, c)$, if $d(c, M) \leq d(a, M)$, with positive probability all players locate on the same side and we reach $A$. If $d(a, M)<d(c, M)$, $B R_{t}=((a-1) * 3, c-1)$. If $d(a-1, M)=d(c, M)$, we are in an absorbing state. Otherwise the maximal endpoint has decreased and we are in $\Omega_{2}$.

- Finally if the configuration is $(a, a, c, c)$, without loss of generality $d(a, M) \leq$ $d(c, M)$ and with positive probability, $B R_{t}=(a-1) * 4 \in A$.

Now, consider a date $t$ at which exactly one player is relocated randomly. Let $L_{p}$ and $R_{p}$ be the leftmost and rightmost endpoint at time $t$. At $t+1$, the players who best reply are not further from the center than $\max \left\{-L_{p}-1, R_{p}+1\right\}$. Suppose that $B R_{t+1}=(a, b, c, r)$. The player who locates at random at $r$ locates outside the interval $I$. At $t+2$, the player located at random will locate at $a-1$ or $c+1$ and the remaining players at $a-1$ if $r$ is to the right and at $c+1$ if $r$ is to the left. Thus at $t+2$, no player locates further away than $\max \{-a-2, d+2\}$.

When exactly one player relocates at random, each player is chosen to be the one who does with probability $\frac{1}{4}$. His probability of being on the right/left side respectively is $\frac{1}{2}$.

Let us show that the probability of entering $A$ is at least $\frac{1}{8}$, in other words there is at least one player whose random relocation leads to $\mathrm{BR}$ in $A$. Let $a, b, c, d$ be (one of) the best replies without random relocation at time $t+1$. Note that now $(a, b, c, d)$ is the configuration we reach by best replies (in the absence of random relocations) and not as before the configuration we start from. It is obvious that if at least three players are on the same side it is sufficient to relocate the last one. Therefore 
suppose $a \leq b<0 \leq c \leq d$. Suppose first that $d(a, M) \leq d(d, M)$ (without loss of generality). Relocate the player whose deterministic best reply was $c$ to the right of $d$ (such a relocation occurs with probability $\frac{1}{8}$ ), so that the best reply with random relocation is $B R_{t+1}=(a, b, d, r)$. Since $r>>d, B R_{t+2}=((a-1) * 3, b-1) \in A$. It is now sufficient to note that whenever we are in $A \bigcap I$, if no random relocation occurs, we reach a configuration of the form $\bar{x} \in I$ in two steps. Therefore, if we reach a state in $A \bigcap I$ in two or three steps starting from $\omega$, we reach a configuration of the form $\bar{x} \in I$ in four or five steps.

We will also need a lemma that tells us that when the sequence is typical, a state of the type $\bar{x} \in I$ leads to $[M-3, M+3]$.

Lemma 6 If we are in $\bar{x} \in I$ at date $t$ and the sequence is typical until $t+l$, we reach a state in $[M-3, M+3]$ within l periods.

Proof: (of Lemma 6) Suppose without loss of generality that $x<M$. If a random relocation outside $I$ occurs, the configuration can be $(r, x * 3)$ and then $B R=\overline{x+1}$. Given that this is the only random relocation in $l$ periods, best replies take us into $[M-3, M+3]$ within $l$ periods. If the configuration with random location is $(x * 3, r)$, $r>M$, then $B R=((x-1) * 3, x+1)$, and since all players are on the same side, again best replies take us into $[M-3, M+3]$ within $l$ periods.

\section{C.3 Best reply dynamics starting from an absorbing class $\Omega_{A C}^{l / 2}$}

We also need to determine what happens when we exit an absorbing class in $\Omega_{A C}^{l / 2}$. 
Lemma 7 Consider a date $t$ at which the deterministic best reply is $\omega=((M-a) *$ $3,(M+a-1)) \in \Omega_{A C}^{l / 2}$ (without loss of generality). Conditioning on the fact that a single random relocation outside of $I$ occurs at $t$, and no random relocation occurs at $t+1$, the state at $t+1$ can be

- $\omega_{t+1}=((M-a) * 3,(M+a-1)) \in \Omega_{A C}^{l / 2}$, i.e. we remain in the same absorbing class at the same distance from the center.

- $\omega_{t+1} \in A$. This happens with probability at least $p=\frac{1}{8}$, and consequently, the probability that $\omega_{t+3}=\bar{x} \in I$ is at least $p=1 / 8$ times the probability that no random relocation occurs at $t+2$ or $t+3$.

- $\omega_{t+1} \in\{(M-a-1) * 3,(M+a)\}$, that is in an absorbing class one step further from the center than before, which occurs with at most probability $1-p$.

Proof: (of Lemma 7) Consider without loss of generality $\omega=((M-a) * 3,(M+$ $a-1)$ ) (the other case is similar by symmetry). If the player who is alone is randomly relocated outside of $I$, if it is on the right we are done because all players are on the same side. This choice has probability $\frac{1}{8}$, as each player is chosen with probability $\frac{1}{4}$, and a location on the right is chosen with probability $\frac{1}{2}$. If it is on the left, the configuration with random relocation is $(r,(M-a) * 3)$ and $B R_{t}=(M-a-1,(M-a+1) * 3) \in A \bigcap I$. If one of the three players on the same side relocates at random on the left, the configuration with random relocation is $(r,(M-a) * 2, M+a-1)$ and $B R_{t}=(M-a+1,(M+a) * 3) \in \Omega_{A C}^{l / 2}$. If one of the three players on the same side relocates at random on the right, the configuration with random relocation is $((M-a) * 2,(M+a-1), r)$, hence $B R_{t}=((M-a-1) * 3,(M+a)) \in \Omega_{A C}$. Note that we are not necessarily in $\Omega_{A C}^{l / 2}$ because we have moved to an absorbing state one step farther from the center. 
Lemma 8 Starting from any state $\omega \in \Omega_{A C}^{l / 2}$, the probability of returning to $S$ before reaching a configuration in $I^{C}$ is greater than $1-O\left(\frac{\epsilon}{l^{2}}\right)$.

Proof: (of Lemma 8) Consider the probability of returning to $S$ from a state in $\Omega_{A C}^{l / 2}$. This probability is minimized when $\left.\omega=\{(M-a) * 3, M+a-1)\right\}$ with $a=l / 2$, the state in $S$ that is the farthest from the center. Let $q$ be the probability that exactly one random relocation occurs and that it leads to $\{(M-a-1) * 3,(M+a)\}$. With probability $1-q-O\left(\epsilon^{2}\right)$ we return to $S$. With probability $q=O(\epsilon)$ we are in $\{(M-a-1) * 3,(M+a)\}$, an absorbing class that is neither in $S$ nor in $I^{C}$. From this class, we can reach $I^{C}$ in two ways. Either by gradually moving from one absorbing class to another one step farther from the center, i. e. repeating $l / 2$ times case 3 in Lemma 7, or we can exit rapidly because of 'atypical' random relocations. Consider the sequence of random variables drawn at times $t, \ldots t+L$, where $t$ is the date of entry in $((M-a-1) * 3,(M+a))$ and $L=: \frac{l^{4}}{\epsilon}$. Let us define three events $E_{1}, E_{2}, E_{3}$ such that if these three events are realized we return to $S$.

- $E_{1}$ : Let $A_{k}$ be the event that there is no random relocation in $I$ at date $t+k$ or $t+k+1$, and at most one random relocation in $I^{C}$ (either at $t+k$ or at $t+k+1$ but not both), and let $E_{1}$ be the event $\left.\bigcap_{k=1}^{k=L-1} A_{k}\right)$.

- $E_{2}$ is the event that there are at least $O(l)$ random relocations in $I^{C}$.

- $E_{3}$ is the event that at least one random relocation in $I^{C}$ leads to a state $\bar{x} \in I$.

If the three events above are realized, we return to $S$. Consequently the probability that we do not return to $S$ is bounded by $P\left(E_{1}^{C} \cup E_{2}^{C} \cup E_{3}^{C}\right) \leq$ $P\left(E_{1}^{C}\right)+P\left(\left(E_{2}^{C} \bigcup E_{3}^{C}\right)\right)$.

We begin by bounding $P\left(E_{1}\right)$. At each date, the probability that there is no random relocation is $(1-\epsilon)^{4}=: 1-\pi$, with $\pi=4 \epsilon+O\left(\epsilon_{N}^{2}\right)$. The probability that exactly one random relocation occurs at a given date is $q=: 4 \epsilon(1-\epsilon)^{3}=$ 
$4 \epsilon+O\left(\epsilon^{2}\right)$. The probability that the required property is satisified by $A_{1}$ is thus $(1-\pi)^{2}+2(1-\pi) q(N-l) / N=1-O\left(\frac{l \epsilon}{N}\right)$. Either we have no random relocations in $A_{1}$ or there is a random relocation outside of $I$ at one of the dates and no random relocation at the other date. The probability that $A_{k}$ satisfies the property is not independent of the probability that it is satisfied by $A_{k-1}$ since there is one date in common. However the probability $P\left(\bigcap_{k=1}^{k=L-1} A_{k}\right)=$ $\prod_{k=2}^{k=L-1} P\left(A_{k} \mid A_{k-1}\right) P\left(A_{1}\right)$. The conditional probability $P\left(A_{k} \mid A_{k-1}\right)$ is not smaller than $P\left(A_{k}\right)$. Indeed the dependence occurs through the common location $k$ and the probability of a random relocation at this date is lower conditioning on $A_{k-1}$. We have $P\left(\bigcap_{k=1}^{k=L-1} A_{k}\right) \geq\left(1-O\left(\frac{l \epsilon}{N}\right)\right)^{L}=\left(1-O\left(\frac{l \epsilon}{N}\right)\right)^{\left(\frac{N}{l \epsilon}\right)\left(\frac{l^{5}}{N)}\right.}=\exp -O\left(\frac{l^{5}}{N}\right)=1-O\left(\frac{l^{5}}{N}\right)$. Thus $P\left(E_{1}^{C}\right)=O\left(\frac{l^{5}}{N}\right)<O\left(\frac{1}{l^{2}}\right)$ since $\lim _{N \rightarrow \infty} \frac{l^{7}}{N}=0$.

Next, we prove that with high probability the number of random relocations in $I^{C}$ is approximately $L \epsilon$, their expected value. The number of periods at which we obtain random relocations is given by a binomial random variable $\operatorname{Bin}(q, L)$. This variable has mean $q L=O\left(l^{4}\right)$ and standard deviation $\sigma=\sqrt{L q(1-q)}=O\left(l^{2}\right)$. By the Chebycheff inequality, the probability $P(|\operatorname{Bin}(q, L)-q L| \geq k \sigma) \leq \frac{1}{k^{2}}$. Taking for example $k=l$, with probability at least $1-\frac{1}{l^{2}}$, the number of random relocations is $O\left(l^{4}\right)$. Thus, $P\left(E_{2}\right) \geq 1-\frac{1}{l^{2}}$.

Finally, each time a random relocation occurs, it is such that we enter $A$ with probability $p=\frac{1}{8}$, and if no random relocation occurs in the next two steps, which has a probability close to 1 , we then reach $\bar{x} \in I$. Consequently, at each random relocation, the probability of not reaching $\bar{x} \in I$ is smaller than, say, $\frac{8}{9}$. If we condition on the fact that there are $O\left(l^{4}\right)$ random relocations in $I^{C}$, the probability that none leads to $\bar{x} \in I$ is $P\left(E_{3}^{C} \mid E_{2}\right)=\left(\frac{8}{9}\right)^{O\left(l^{4}\right)}<O\left(\epsilon^{2}\right)$ (as a result of Condition 1). Therefore $P\left(\left(E_{2}^{C} \cup E_{3}^{C}\right)\right)=1-P\left(E_{2} \bigcap E_{3}\right)=1-P\left(E_{3} \mid E_{2}\right) P\left(E_{2}\right)=$ $1-\left[1-P\left(E_{3}^{C} \mid E_{2}\right)\right] P\left(E_{2}\right) \leq 1-\left[1-O\left(\epsilon^{2}\right)\right]\left(1-\frac{1}{l^{2}}\right)=O\left(\frac{1}{l^{2}}\right)$

Thus, we conclude that $P\left(E_{1}^{C} \cup E_{2}^{C} \cup E_{3}^{C}\right)=O\left(\frac{1}{l^{2}}\right)$. Since the probability of 
entering $((M-a-1) * 3,(M+a))$ instead of returning to $S$ immediately is $q$, the probability of not returning to $S$ is $O\left(\frac{q}{l^{2}}\right)=O\left(\frac{\epsilon}{l^{2}}\right)$.

Now we can prove Proposition 4.

\section{C.4 Proof of Proposition 4}

We want to bound the probability of returning to $S$ from a state in $S=[M-3, M+$ 3] $\bigcup \Omega_{A C}^{l / 2}$. First, consider the case where we start from an $\omega \in[M-3, M+3]$. Let us bound the probability of returning in at most $l / 4$ periods, assuming that the sequence is typical over these periods. We can assume that we do not enter an absorbing class because if we do we have returned to $S$, so $\left|\Omega_{4}\right|=0$. Note that by Lemma 5 , we have $\left|\Omega_{3}\right|=\left|\Omega_{1}\right|+\left|\Omega_{2}\right|$ since any successor of an element in $\Omega_{3}$ is in $\Omega_{1} \cup \Omega_{2}$. Also note that if $\left|\Omega_{1}\right|<2\left|\Omega_{2}\right|$, then we have returned to the center $M$ because the configurations in $\Omega_{2}$ contract one step and those in $\Omega_{1}$ grow at most two steps. Therefore we must have $\left|\Omega_{3}\right|+\left|\Omega_{1}\right|+\left|\Omega_{2}\right|=\frac{l}{4}$, where $\left|\Omega_{1}\right| \geq 2\left|\Omega_{2}\right|$. Suppose that $\left|\Omega_{1}\right|<\frac{l}{24}$. Then $\left|\Omega_{2}\right| \leq \frac{2 l}{24}$ and $\left|\Omega_{3}\right|<\frac{3 l}{24}$. But this contradicts $\left|\Omega_{3}\right|+\left|\Omega_{1}\right|+\left|\Omega_{2}\right|=\frac{l}{4}$. Consequently, $\left|\Omega_{1}\right| \geq \frac{l}{24}$. Each time we are in $\Omega_{1}$, we enter a state $\bar{x} \in I$ with probability $p$. From such a state we then return to $[M-3, M+3]$ if the sequence is typical by Lemma 6 . The probability of not reaching a $\bar{x} \in I$ in $l / 24$ trials is $(1-p)^{l / 24} \leq O\left(\epsilon^{2}\right)$, which is a consequence of Condition 1 since $0<(1-p)^{\frac{1}{24}}<1$. Consequently, the probability of returning to $\mathrm{S}$ is bounded by $1-(1-p)^{l / 24}$ times the probability that the sequence is typical for $l / 4+l$ periods. Using Lemma 4 this probability is greater than $\left(1-O\left(\epsilon^{2}\right)\right)\left[1-\epsilon^{2} l^{2}\right]=1-O\left(\epsilon^{2} l^{2}\right)$. In the case which was not considered before, where we start from a state $\omega \in \Omega_{A C}^{l / 2}$, it is sufficient to apply Lemma 8. 


\section{C.5 Proof of Proposition 5}

Proposition 5 is a consequence of Lemma 9 below

Lemma 9 For any configuration $\omega \in A^{C}$, there exists a random relocation of a player, which occurs with probability $O(\epsilon)$ such that $A$ is reached in at most three steps.

Proof: The proof is based on a number of lemmas dealing with all possible cases of configurations in $A^{C}$.

The first lemma deals with configurations where three players are on the same side:

Lemma 10 Suppose that $\omega$ is a configuration such that $a \leq b \leq c<M<d$. Then either $B R(\omega)$ is a configuration such that $a \leq b<M<c \leq d$, or a single random relocation will place $B R(\omega)$ in $A$.

Proof: Indeed, suppose that the deterministic best replies place three players on the same side, it is then sufficient to relocate at random the last player. If he is relocated on the same side as the others, which occurs with probability $\frac{1}{2}$, we are in A.

Therefore, from now on, we restrict attention to configurations with two players on each side of the center: $a \leq b<M<c \leq d$.

In Lemma 11 below, we consider the case where both endpoints of the configuration are close to the center so that no player has an interior best reply.

Lemma 11 Suppose that $\max \{d(a, M), d(d, M)\}<\frac{N}{4}$, then the set $A$ is reached within at most two steps with a probability at least equal to $\epsilon c_{1}$, where $c_{1}$ is a constant independent of $N$. 
Proof: If $\max \{d(a, M), d(d, M)\}<\frac{N}{4}$, the interior players prefer to relocate at the endpoints. We have $a \leq b<M<c \leq d$, and $d(d, M) \leq d(a, M)$ (without loss of generality), then with probability at least $\frac{1}{4}$ both interior players locate at $d+1$, and the player at $d$ locates at $c+1$. It is sufficient to randomly relocate the player at $a$ on the right side to be in $A$.

When the endpoints of a configuration are far from the center, best replies can be interior. We summarize some useful properties of interior best replies. We denote by $U_{[a, b]}$ a discrete uniform law on the set of locations strictly between $a$ and $b$.

\section{Property 1}

- If the players at a or d prefer an interior location it is given by $U_{[b, c]}$

- If the player at $b$ has an interior best reply, it is given by the uniform random variable $U_{[a, c]}$ or $U_{[c, d]}$

- If the player at c has an interior best reply, it is given by the uniform random variable $U_{[b, d]}$ or $U_{[a, b]}$

- If the best reply of the player at $b$ is $U_{[c, d]}$, the best reply of the player at $c$ is $U_{[b, d]}$

- If the best reply of the player at $c$ is $U_{[a, b]}$, the best reply of the player at a is $U_{[a, c]}$

Lemma 12 Suppose that $U \in\left\{U_{[a, c]}, U_{[b, c]}, U_{[b, d]}, U_{[c, d]}, U_{[a, b]}\right\}$ is an interior best reply of some player, then $P\left(d(U, M)<\frac{N}{4}\right) \geq c_{2}$, where $c_{2}>0$ is a constant independent of $N$.

Proof: For uniform variables on the intervals $[a, c],[b, c]$ and $[b, d]$, the result is obvious since these intervals contain $M$. For example $[a, c]=[a, M] \bigcup(M, c]$ and at 
least one of the two intervals must have a strictly positie probability to make $[a, c]$ a best reply, and similarly for $[b, c]$ and $[b, d]$. Let us show that the best reply $U_{[c, d]}$ (and similarly $U_{[a, b]}$ by symetry ) can only occur if $d(c, M) \leq\left[\frac{1}{4}-K\right] N$, implying $P\left(d\left(U_{[c, d]}, M\right)<\frac{N}{4}\right) \geq K$. If $d(c, M)>\left[\frac{1}{4}-K\right] N$, the utility of location $U_{[c, d]}$ is inferior to $N / 8+K N$, but then if $d(a, M)<\frac{N}{4}$, the player at $b$ prefers location $a-1$, which gives a utility of at least $\frac{N}{4}$. And if $d(a, M)>\frac{N}{4}$, he prefers $U_{[a, c]}$ which provides a utility superior to $\frac{N}{4}-K N$ (with $K$ assumed small but positive). Thus, $U_{[c, d]}$ cannot be a best reply.

Lemma 13 Let $c_{3}=\frac{1}{10}$. If $d(b, M)>k N$ and $d(c, M)>c_{3} N$, then any configuration $\omega$ where at least one player has an interior best reply reaches $A$ with a single random relocation.

Proof: Suppose at least one player has an interior best reply $U \in$ $\left\{U_{[a, c]}, U_{[b, c]}, U_{[b, d]}\right\}$. By the assumptions, $P(U>M)>\frac{1}{10}$ and $P(U<M)>\frac{1}{10}$. Such a player can end up on any side of the center with positive probability. If one of the remaining three players is alone on his side, it suffices to relocate him at random. We note that by Property 1 , if the interior reply (of player $b$ ) is $U_{[c, d]}$, then the best reply of the player at $c$ is $U_{[b, d]}$, and similarly if the interior reply (of player $c)$ is $U_{[a, b]}$ then that of player $b$ is $U_{[a, c]}$.

The cases that were not covered by Lemma 13 are covered by Lemma 14 .

Lemma 14 If $d(b, M) \leq c_{3} N$ or $d(c, M) \leq c_{3} N$, where $c_{3}=\frac{1}{10}$, then any configuration $\omega$ reaches $A$ in at most three steps involving a single random relocation.

Proof: Suppose without loss of generality $d(b, M) \leq d(c, M)$. 
(i) Suppose $d(a, M)>\frac{N}{4}$ and $d(d, M)<\frac{N}{4}$. Since the point $d+1 \leq \frac{N}{4}$ is available, no player locates at $a-1$. If some player has an interior best reply, by lemma 12 , the realized location is closer than $\frac{N}{4}$ to the center with probability at least $\frac{1}{4}$, and we reach a configuration where both endpoints are inferior to $\frac{N}{4}$, a case already treated in Lemma 11.

(ii) The case $d(a, M)<\frac{N}{4}$ and $d(d, M)>\frac{N}{4}$ is the same as the one above, by symmetry, since the positions of $b$ and $d$ did not intervene in the argument above.

(iii) Suppose $d(a, M)>\frac{N}{4}$ and $d(d, M)>\frac{N}{4}$.

(a) If $d(b, M) \leq d(c, M)<c_{3} N$, then the best replies are $b-1, c+1$ and the interior players either locate at the smallest endpoint or in the interior. In both cases, with positive probability, the smallest endpoint in $B R_{t}(\omega)$ is closer than $\frac{N}{4}$ to the center, and we are in one of the cases considered above.

(b) Suppose $d(c, M) \geq c_{3} N$, and $d(a, M)>d(d, M)$. If the best reply of the player at $b$ is $U_{[a, c]}$, he has positive probability of ending up on either side. We can then apply the argument in the proof of Lemma 13: at least two of the other players locate on the same side. Relocate the remaining one on this side. Thus suppose the player at $b$ locates at $d+1$ or at $U_{[c, d]}$. The players at $c$ and $d$ locate on the right with positive probability. ${ }^{30}$ The player at $a$ relocates at $b-1$. It is sufficient to relocate the player whose best reply is $b-1$ on the right side.

(c) Suppose $d(c, M) \geq c_{3} N$, and $d(a, M) \leq d(d, M)$. If the best reply of the player at $b$ is $U_{[a, c]}$, he has positive probability of ending up on either

\footnotetext{
$b$.

${ }^{30}$ Indeed $U_{[a, b]}$ is not a best reply for the player at $c$ if $U_{[a, c]}$ is not a best reply for the player at
} 
side, and we are done by the argument in the proof of Lemma 13. Thus, suppose the player at $b$ locates at $a-1$ or $U_{[c, d]}$ and the player at $a$ relocates at $b-1$. Note that $d(c, M)<N / 5$, because otherwise the player at $b$ would prefer the interval $[a, c]$. The possible locations to the right of $c$ are $U_{[b, d]}$ and $U_{[c, d]}$ but $P\left(d\left(U_{[c, d]}, M\right)<\frac{N}{4}\right) \geq \frac{N / 4-N / 5}{d-c}$. With positive probability, we reach a configuration whose rightmost endpoint is smaller than $\frac{N}{4}$, a case that has been analyzed before.

Combining Lemma 10 to 14 proves Lemma 9.

Proposition 5 results almost immediately from this lemma. Indeed, once in $A$, the configuration will move towards the center at the pace of one location per time step. To reach the center, it is thus sufficient that no random relocation occurs in $\mathrm{N}$ steps. The probability that this is the case is $(1-p)^{N}$, where $p=1-(1-\epsilon)^{4}$. Since $\epsilon<1 / N$, we have $(1-p)^{N}=O(1)$. Consequently, the probability of reaching $\mathrm{M}$ from $b \in A^{C}$ is given by the probability of entering $A$, which is $O(\epsilon)$, since a single random relocation takes us to $A$, times the probability that no random relocation occurs for $N$ periods after entering $A$, which has probability $O(1)$. The result in Proposition 5 follows.

\section{Appendix: Stochastic Stability of Nash Equilibria}

Proof: (of Theorem 3) Let $\left(L_{i}\right)_{i=1}^{i=r}$ be the locations of the players at date $t$, with the convention that $L_{1}$ and $L_{r}$ are the leftmost and rightmost locations. By abuse of notation, if two players are (non-exactly) paired, we will refer to their location 
as $L_{i}$ if the leftmost player in the pair is located at $L_{i}$ and the rightmost one in the pair at $L_{i}+1$. Let $i=: \operatorname{argmax} L_{i+3}-L_{i}$. This quantity is well defined if the players are located in at least four different locations. This will always be true if $n \geq 7$. If $n=6$ it is true if all the players are not paired in equilibrium. We will assume that this holds and the cases $n=4,5$ or $n=6$ with all players paired will be analyzed separately. Suppose first that the players at $L_{i+1}$ and $L_{i+2}$ are alone or exactly paired. The player/players at $L_{i+1}$ are indifferent between their current location and $L_{i}+1$. The player/players at $L_{i+2}$ are indifferent between their current location and $L_{i+3}-1$, so that if the players relocate at $L_{i}+1$ and $L_{i+3}-1$, then in the next period, a player can gain $\left(L_{i+3}-1-\left(L_{i}+1\right)\right) / 2$ by locating anywhere in $\left[L_{i}+2, L_{i+3}-2\right]$. Any player $j$ who is not in this interval and who remains at his current position $L_{j}$ gains $\left(L_{j+1}-L_{j-1}\right) / 2<\left(L_{i+3}-1-\left(L_{i}+1\right)\right) / 2$ since $i$ was chosen to have for all j $L_{i+3}-L_{i} \geq L_{j+2}-L_{j-1}=L_{j+2}-L_{j+1}+L_{j+1}-L_{j-1}$ and $L_{j+2}-L_{j+1}>>1$ for every $j$. Therefore, for every player, every location in $\left[L_{i}+2, L_{i+3}-2\right]$ is a best reply.

Suppose instead that the players at $L_{i+1}$ and $L_{i+2}$ are (non-exactly) paired. In this case, it is not possible to obtain a large empty interval in one step and we need to move players in several steps. The locations chosen amont the possible best replies in the first step are picked in order to obtain new empty intervals of suitable sizes. At time $t$, by indifference, with positive probability, the leftmost player at $L_{i+1}$ can move to $L_{i}+1$, the rightmost player at $L_{i+2}$ to $L_{i+3}-3$ and the leftmost player at $L_{i+2}$ to $L_{i+2}-2$. With positive probability, the occupied locations in $\left[L_{i}, L_{i+3}\right]$ at $t+1$ will then be $\left\{L_{i}, L_{i}+1, L_{i+1}+1, L_{i+2}-2, L_{i+3}-3, L_{i+3}\right\}$. The unoccupied intervals between $L_{i}$ and $L_{i+3}$ are $\left[L_{i}+2, L_{i+1}\right],\left[L_{i+1}+2, L_{i+2}-3\right]$, $\left[L_{i+2}-1, L_{i+3}-4\right]$. If we compare the sizes of these unoccupied intervals with the sizes of the unoccupied intervals in Nash equilibrium prior to the moves at time $t$ we have $L_{i+1}-\left(L_{i}+2\right)=\left(L_{i+1}-1\right)-\left(L_{i}+1\right),\left(L_{i+2}-3\right)-\left(L_{i+1}+2\right)=\left(L_{i+2}-1\right)-\left(L_{i+1}+2\right)-2$ 
and $\left(L_{i+3}-4\right)-\left(L_{i+2}-1\right)=\left(L_{i+3}-1\right)-\left(L_{i+2}+2\right)$. The moves at $t$ have reduced the middle market by two, while conserving the sizes of the two others. By property (v), the size of the middle market in Nash equilibrium cannot exceed the size of the left and right markets by more than one. Therefore, at $t+1$, the middle interval cannot be strictly preferred to the left and right ones. The markets of the players located at $L_{i}$ and $L_{i+3}$ have been reduced and they might want to move. If interval $\left[L_{i}+2, L_{i+1}\right]$ is a best reply for the players located at $L_{i}$ and $L_{i+3}, L_{i}+2$ is a possible location and if $\left[L_{i+2}-1, L_{i+3}-4\right]$ is a best reply, $L_{i+3}-4$ is a possible location. If none of these intervals are a best reply, the best reply for these players is either to stay where they are or relocate elsewhere (we note that if they relocate elsewhere, their presence will only have for effect to reduce the markets of the players where they relocate). It is also important to note that since the sizes of the different markets between $L_{i}$ and $L_{i+3}$ have not increased compared to their sizes in Nash equilibrium, no other players want to move into these markets at this time. As for the players at $L_{i+1}+1$ and $L_{i+2}-2$, by indifference, they can move to $L_{i}+2$ and $L_{i+3}-4$ respectively at $t+1$, while the players at $L_{i}+1$ and $L_{i+3}-3$ stay where they are.

At $t+2$, with positive probability, we have thus reached a configuration where the interval $\left[L_{i}+3, L_{i+3}-5\right]$ is empty. Similarly to the first case, a player can gain $\left(L_{i+3}-L_{i}-8\right) / 2$ by locating anywhere in this interval. If he remains at his current position $L_{j}$, he gains at most $\left(L_{j+1}-L_{j-1}\right) / 2<\left(L_{i+3}-L_{i}-8\right) / 2$ for every $j$. Therefore, for every player, every location in $\left[L_{i}+3, L_{i+3}-5\right]$ is a best reply.

Finally, if the players at $L_{i+1}$ (without loss of generality) are non exactly paired and those at $L_{i+2}$ are exactly paired, at $t$, by indifference, one of the players at $L_{i+2}$ can move to $L_{i+3}-3$ and the other one to $L_{i+2}-2$, the leftmost player at $L_{i+1}$ can move to $L_{i}+2$ and the rightmost player stay where he is. This configuration is similar to the one reached at time $t$ in the case analyzed previously and by the 
same arguments, at $t+2$, with positive probability locations in $\left[L_{i}+3, L_{i+3}-4\right]$ are best replies.

If the whole interval $\left[L_{i}+2, L_{i+3}-2\right]$ is not on the same side of the center, the probability of locating in say $\{1, \ldots, M\} \bigcap\left[L_{i}+2, L_{i+3}-2\right]$ is positive (and similarly for $\{1, \ldots, M\} \bigcap\left[L_{i}+3, L_{i+3}-5\right]$ etc). Thus, there is a positive probability of reaching a configuration where all players are on the same side of the center (i.e. a configuration in $A$ ). Therefore, in any Nash equilibrium, with positive probability, we reach, in at most three steps, a state where all players are on the same side of the center. We have already seen that from such a state we reach the center with probability 1 and from the center, there is a positive probability of reaching some absorbing state in $\Omega_{A C}$.

We will now analyze the cases $n=4,5$ and $n=6$ with all players paired. In all these cases, the proof just consist in showing that we have a positive probability of a best reply sequence leading to a configuration where all players are on the same side. In the cases $n=4,5,6$, there are always three possible sub-cases depending on whether the peripheral players are exactly or not exactly paired: Case (a): all the peripheral players are exactly matched and located at $l$ and $r$ respectively. Case (b): the peripheral players on the left (without loss of generality) are not exactly paired and located at $l, l+1$ and the rightmost players are exactly paired and located at $r$. finally, in case (c), all the peripheral players can be non exactly paired and located at $l, l+1$ and $r, r+1$. We should also note that it is possible that some of the Nash equilibria that we analyze do not always exist because the inequalities that must hold to ensure their stability are not verified for all $N$. However, it is easier to prove that if the equilibrium exists the inequalities defining it lead to instability than to check its existence.

- $n=4$ :

Case (a). The initial locations are $(l, l, r, r)$. Assume that the players at $l$ do 
not want to deviate to $l-1$ and strictly prefer their current location to $l+1$ (or any other interior location). These conditions imply that $l \leq \frac{l+1}{2}+\frac{r-l-1}{4}$ and $\frac{r-l}{2}<\frac{l+1}{2}+\frac{r-l-1}{4}$. Rewriting these conditions gives us $3 l \leq r+1$ and $3 l>r-1$. Thus $3 l \in\{r, r+1\}$. Suppose first that $3 l=r+1$. Assuming that the players at $r$ strictly prefer $r$ to $l-1$, we have $l<\frac{r-l-1}{4}+\frac{N-r+1}{2} \Longleftrightarrow$ $5 l<2 N-r+1 \Longleftrightarrow N>4 l-1$, where the last inequality comes from $3 l=r+1$. The players at $r$ must prefer their current location to $r+1$, which gives $\frac{r-l-1}{4}+\frac{N-r+1}{2} \geq N-r \Longleftrightarrow 2 N \leq 3 r-l+1 \Longleftrightarrow N \leq 4 l-1$. Together we have $4 l-1<N \leq 4 l-1$, which is impossible. The weak inequalities must be verified, for Nash equilibrium to hold. Therefore it must be that some of the equalities that were assumed to be strict are not. Thus, either the players at $l$ are indifferent between $l$ and an interior location, or the players at $r$ are indifferent between $r$ and $l-1$. In both cases, due to indifference, we can move two players to the same side as the the players on the opposite side and we are done. Suppose $3 l=r$. The players at $r$ must not want to move to $l-1$, which gives $\frac{r-l-1}{4}+\frac{N-r+1}{2}>l \Longleftrightarrow 2 N>8 l-1$. The players at $l$ must not want to move to $r+1$, which gives $N-r \leq \frac{l+1}{2}+\frac{r-l-1}{4} \Longleftrightarrow 4 N \leq$ $5 r+l+1 \Longleftrightarrow 4 N \leq 16 l+1$. Thus we must have $16 l-2<4 N \leq 16 l+1$. Since $l$ is a natural number, we must have $N=4 l$. If the number of locations is a multiple of 4 , we can have a Nash equilibrium with 4 players that is not weak. This does not necessarily imply that the Nash equilibrium belongs to the stochastically stable steady states but we cannot apply the same types of arguments as when the equilibrium is weak. This case is excluded from our analysis.

Case (b): The initial configuration is $(l, l+1, r, r)$. Relocate the player at $l+1$ who is indifferent between interior locations to $M-4$, to obtain configuration $(l, M-4, r, r)$. The player at $l$ has $M-5$ for best reply and the player at 
$M-4$ is indifferent between interior locations in $[l, r]$. The left market of the players at $r$ has been reduced significantly. Unless their unique best reply is $r+1$, with positive probability, all players can end up on the same side. If their unique best reply is $r+1,(M-5, M-4, r+1, r+1) \in B R(l, M-4, r, r)$ and $(3 *(M-6), M-5) \in B R_{2}$ and so in two steps, with positive probability, all players can end up on the same side.

Case (c) : the initial configuration is $(l, l+1, r, r+1)$. Suppose without loss of generality that $l \geq N-(r+1)$ so that location $l-1$ is prefered to $r+2$. Then $(l, l+1, M-2, r+1) \in B R(l, l+1, r, r+1)$ and $(l-1, l, M-2, M-1) \in B R_{2}$.

- $n=5$ :

We note the middle player's location by $m$. In all the Nash equilibria, we have some inequalities that must be verified. It is necessary to have $\frac{r-l}{2} \geq \max \{l, N-r-1\}$ because the middle player must not want to deviate to location $l-1$ or to $r+2$. Moreover, we must have $\frac{m-l}{2} \geq l$ to ensure that none of the left-peripheral players want to deviate to $l-1$. Indeed, if the leftperipheral players are exactly paired, they earn $\frac{l+1}{2}+\frac{m-l-1}{4}$. They must not want to deviate to location $l-1$ implying that $l \leq \frac{l+1}{2}+\frac{m-l-1}{4} \leftrightarrow 2 l \leq m-l+1$. If the left-most players are non-exactly paired, the player at $l+1$ who earns $\frac{m-l}{2}$ at his current location must not want to deviate to $l-1$. A necessary condition is that $\frac{m-l}{2} \geq l$. Taking the weakest condition resulting from the two possible cases we have $2 l \leq m-l+1$ and by similar reasoning for the rightmost players we must have $\frac{r-m}{2} \geq N-r+1$. To summarize, in any Nash equilibrium, we must have $2 l \leq m-l+1$ and $2(N-r) \leq r-m+1$.

Case (a): Let $(l, l, m, r, r)$ be the initial configuration at $t=0$. By indifference of the middle player, $(l, l, l+1, r, r) \in B R(l, l, m, r, r)$. At $t=2$, the payoff for a location inside $[l+2, r-1]$ is $\frac{(r-1)-(l+2)}{2}=\frac{(r-m)+(m-l)-3}{2}>>\max \left\{N-r, \frac{l}{2}\right\}$. 
Therefore the leftmost players prefer a location in $[l+2, r-1]$. If the rightmost players also prefer an interior location we are done because with positive probability, all players pick a location in $[l+2, r-1]$ on the left side of the center. If not, at $t=2$, locate one of the leftmost players at $l+2$ and the other one at $r-1$. This reduces the left market of the rightmost players enough to make them want to move. At $t+3$, all players prefer a location in $[l+3, r-2]$. With positive probability, all player pick a location on the left of the center.

Case (b): The initial configuration is $(l, l+1, m, r, r)$. By indifference of the middle player, $(l, l+1, l+2, r, r) \in B R(l, l+1, m, r, r)$. As in (a), at $t+2$, the two leftmost players prefer a location in $[l+3, r-1]$. The rest of the argument is analogous to case (a). Case (c): The initial configuration is $(l, l+1, m, r, r+1)$. By indifference of the middle player, $(l, l+1, l+2, r, r+1) \in$ $B R(l, l+1, m, r, r+1)$. As in (a), at time $t=2$, the two leftmost players prefer a location in $[l+3, r-1]$. With positive probability they locate at $l+3$. The player at $r$ can locate at $l+3$ by indifference, so $((l+3) * 3, r+1) \in B R_{2}$. At $t+3$, the player at $r+1$ moves to $l+4$. All other players prefer a location in $[l+4, r]$. Thus, with positive probability, all player pick a location on the left of the center.

- $n=6$ :

The middle players, exactly or non exactly paired must not want to deviate, implying that $\min \left\{\frac{m+1-l}{2}, \frac{r-m}{2}, \frac{r-(l+1)}{4}\right\} \geq \max \{l, N-r\}$.

Case (a): The initial configuration is $(l, l, m, m, r, r)$ or $(l, l, m, m+1, r, r)$. By indifference, at a time $t=1$, the two middle players, whether they are exactly or not exactly paired, can relocate at $l+1$ and $r-1$, so as to obtain configuration $(l, l, l+1, r-1, r, r)$. At $t=2$, the payoff from locating inside $[l+2, r-2]$ is $\frac{(r-2)-(l+2)}{2}=\frac{(r-m)+(m-l)-4}{2}>\max \left\{\frac{N-r+1}{2}, \frac{l+1}{2}\right\}$. Therefore the 
players at $l$ and $r$ both prefer a location inside $[l+2, r-2]$ to their current location. The player at $r-1$ is indifferent between this location and other locations in $[l+2, r-2]$. Thus, with positive probability, at $t+1$, they all locate on the same side of the center.

Case (b): The initial configuration is $(l, l+1, m, m, r, r)$ or $(l, l+1, m, m+$ $1, r, r)$. By indifference, at a time $t=1$, the two middle players, whether they are exactly or not exactly paired, can relocate at $l+2$ and $r-1$, so as to obtain configuration $(l, l+1, l+2, r-1, r, r)$. At $t=2$, similarly to case (a), the players at $l$ and $l+1$ prefer a location inside $[l+3 r-2]$ and the same for the players at $r$. The player at $r-1$ is indifferent between all locations in $[l+3, r-1]$. With positive probability, at $t=2$, all players locate on the same side of the center. Case (c): The initial configuration is $(l, l+1, m, m, r, r+1)$ or $(l, l+1, m, m+1, r, r+1)$. By indifference, at a time $t=1$, the two middle players can relocate at $l+2$ and $r-1$, so as to obtain configuration $(l, l+1, l+2, r-1, r, r+1)$. At $t=2$, as in case (a), the players at $l, l+1, r$ and $r+1$ prefer locations inside $[l+3, r-2]$, the players at $l+2$ and $r-1$ are indifferent between interior locations. With positive probability, at $t=2$, all players locate on the same side of the center. 